\title{
Synthetic representation of the Galilean satellites' orbital motions from L1 ephemerides *
}

\author{
V. Lainey ${ }^{1,3}$, L. Duriez ${ }^{2,3}$, and A. Vienne $e^{2,3}$ \\ 1 Royal Observatory of Belgium, 3 avenue Circulaire, 1180 Bruxelles, Belgium \\ e-mail: Lainey@oma . be \\ 2 Université Lille1 IMCCE/LAL, 1 impasse de l'Observatoire, 59000 Lille, France \\ 3 Institut de mécanique céleste et de calcul des éphémérides-Observatoire de Paris, UMR 8028 du CNRS, \\ 77 avenue Denfert-Rochereau, 75014 Paris, France
}

Received 31 January 2006 / Accepted 10 April 2006

ABSTRACT

\begin{abstract}
Aims. We aim to provide a synthetic representation of the orbital motion of Io, Europa, Ganymede, and Callisto. It corresponds to a quasi-periodic Fourier series whose arguments are integer combinations of the fundamental arguments of the dynamical system, which are also given.

Methods. The present series are issued from frequency analysis and digital filtering treatments. The initial samplings have been taken from a former numerical integration fit to the observations and referenced as L1 ephemerides.

Results. Our representation allows for the identification of all the significant perturbations in the system, which helps us to understand these orbital motions. It is also useful in other related topics: the dynamical history of the system and studies requiring us to extract only major terms to compute motions such as the rotation of the bodies.
\end{abstract}

Key words. ephemerides - celestrial mechanics - planets and satellites: general

\section{Introduction}

To have very accurate ephemerides of the Galilean satellites Io, Europa, Ganymede, and Callisto, we built a new dynamical model leading to numerical simulations of their motions (Lainey et al. 2004a). This model has been adjusted to different kinds of observations (photographic, CCD, and mutual events) done between 1891 and 2003. Thousands of observations have been used, including the most accurate ones, issued from the mutual event campaigns (PHEMU) organized by the IMCCE, from 1985 to 1992 . As a result, we have given a numerical representation that is able to produce ephemerides (Lainey et al. 2004b), referenced here as L1 ephemerides.

A numerical integration of the adjusted model was performed over 1700 years. Instead of using a numerical representation by Tchebychev polynomials, as is commonly made to interpolate between time steps, we preferred using a frequency analysis to build a quasi-periodic representation of the motions. This method was first successfully used by Carpino et al. (1987) in the case of planetary motions. Our representation has today a faithful accuracy of a few tens or so kilometers upon one century and remains definite upon more than 1700 years.

Furthermore, this synthetic form is fundamental for understanding the dynamics of the system. For example, the analytical representation of the eight main Saturnian satellites' motion (Vienne \& Duriez 1995) has allowed us to discover new terms. These terms lead to a better knowledge of the evolution of the Mimas-Tethys resonance (Champenois \& Vienne 1999a,b). For the Galilean satellites, a representation rather similar to the one

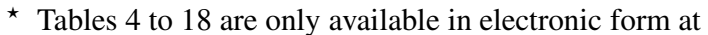
http://www.edpsciences.org presented here has been used in the works of Henrard (2005a,b) dealing with the rotation of Europa and Io. The present work has also been used by Noyelles (2005) to describe the evolution of the Galilean system. More generally, a synthetic form helps to identify and understand the origin and effects of the perturbations that occur in the motion of the corresponding satellites.

At the end of the 70s, Lieske (1977) refreshed Sampson analytical ephemerides (Sampson 1921) for use in Voyager spacecraft navigation. Despite the important works done by several authors (e.g. Sagnier 1975; Vu 1982; Thuillot 1984), these ephemerides are still probably the only ones that have both an analytical model and a fit to the observations. However, the related representation suffer from a few inconsistencies: the number of parameters to fit was higher than the number of degrees of freedom, and the Laplacian libration was artificially added in the series. Recently, Lainey et al. (2004a) also pointed out that some long period terms were missing in Sampson-Lieske theory. Moreover, the representation was developed in cylindrical variables instead of the usual elliptical elements. Hence, comparison between the present work and the Sampson-Lieske series is rather difficult. The present paper provides a representation of the Galilean system. We have avoided most difficulties encountered by Sampson (1921) and Lieske (1977), thanks to numerical integration. Because the numerical integration used to simulate the satellite motions had previously been fit to the observations, its present synthetic representation can be used for ephemerides purposes. This synthetic formulation offers the advantage of describing the Galilean system over a very long time span (as long as tidal effects and long solar period terms are considered negligible).

In the present paper, we give the synthetic representation of the solution L1 and describe the method developed to 
obtain it. This representation corresponds to trigonometric series whose arguments are integer combinations of fundamental arguments of the system: $L_{i}$ (the linear parts of mean longitudes of the satellites and Sun), $\varpi_{i}$ and $\Omega_{i}$ (the proper modes of the pericenters and nodes), and $v, \rho$, and $\Psi$ (the arguments of great inequalities and resonances). First, in Sect. 2, we study the frequency spectrum of the Galilean dynamical system and characterize their short and long periods. Section 3 presents how we generated the different samplings we had to analyse. In Sect. 4 the frequency analysis algorithm we used is discussed. The next section presents the filtering technique involved in separating the long period from the short period. The last section is devoted to the synthetic representation.

\section{Form of the spectrum}

A Fourier analysis usually requires a previous spectral knowledge of the signal to determine optimal sample step size and time span. We have used routines from Duriez (1990, 2003) adapted to explore all the significant short period terms. For a given dynamical system of satellites (characterized by the satellite oblateness and mutual perturbations), these routines determine the amplitude of a given inequality $k_{i} L_{i}+k_{j} L_{j}$, where $L_{i}$ and $L_{j}$ are the linear parts of the mean longitudes of the satellites $i$ and $j$, and where $k_{i}$ and $k_{j}$ are integers. We recall that the subscript 1 is for Io, 2 for Europa, 3 for Ganymede, and 4 for Callisto.

The computations are done at the first order with respect to the masses. In these routines, the amplitudes are in fact a function of $z=e \operatorname{expi} \varpi$ and $\zeta=\sin \frac{I}{2} \exp i \Omega$ of each satellite $(e, I$, $\varpi$, and $\Omega$ denoting, respectively, the eccentricity, the inclination, the longitude of the pericenter, and the longitude of the node). Because we only want an estimation of the amplitude of the inequality, we used the maximum values for the eccentricities and the inclinations from Ferraz-Mello (1979). We explored all the inequalities for which $k_{i}$ and $k_{j}$ were lower than 50 and for which $k_{i}+k_{j}$ was lower than 8 (the maximum degree in eccentricityinclination was then 8 because of the d'Alembert rule). We retained all the short period terms with a truncation level of one kilometer. One can find the list of those greater than $10 \mathrm{~km}$ in Lainey (2002). It appeared that the short period term of significant amplitude and longest period corresponds to the inequality $L_{3}-2 L_{4}$ (50,2 days) and affects Ganymede. The significantly shortest short period is higher than 0.6 day.

The previous routines may also be used for the long period terms. Those involve the node and pericenter precessions (proper elements), the great inequalities $L_{1}-2 L_{2}=L_{2}-2 L_{3}+180^{\circ}$ denoted $v$ in the following text, the De Haerdtl inequality $3 L_{3}-7 L_{4}$ denoted $\rho$, the Laplacian libration argument $\Psi$, and the solar Jovicentric motion ${ }^{1}$. However, it was easier to explore these terms by a preliminary frequency analysis of L1 (without the filtering procedure described in Sect. 5.1), showing that no significant long period term exists with a period below 160 days.

\section{Sampling}

To analyse L1 ephemerides, we used exactly the same software, initial conditions and parameter values that were used in Lainey et al. (2004b). The software computes and delivers Cartesian

\footnotetext{
1 As Saturn was also introduced indirectly in L1 modeling by means of the planetary ephemerides DE406, its motion should also be considered here, in particular because of its great inequality in relation to Jupiter. However, no identification introducing such frequency was possible, mainly because of the long period treatment (see Sect. 5.2).
}

positions and velocities of each satellite in a Jovicentric J2000 Earth mean equatorial frame. To obtain all samplings in the usual elliptical elements, we first rotated each set of Cartesian coordinates in a J2000 Jovian equatorial frame. Then, a routine was applied to shift from Cartesian to Keplerian elements. This operation was done for each data point.

\section{Frequency analysis algorithm}

The frequency analysis algorithm we used is very similar to the one used in Vienne \& Duriez (1992). We only indicate the main points here; however, the reader can find more details in the cited paper. First, a FFT is performed upon the initial signal determined by the numerical integration. We retain the frequency corresponding to the highest amplitude from the FFT. This frequency is only known with the precision $v_{0}=\frac{\pi}{T}$, where $T$ is the time span of the numerical integration. $v_{0}$ is called the fundamental frequency. Then, a fine analysis (Laskar 1993 ) is made in order to have the exact value of the frequency. If this frequency is separated enough from the others, the precision reaches $1.24 \sqrt{\epsilon} v_{0}$ (with the use of the Hanning window), where $\epsilon$ is the epsilon-machine precision of the computer. "Enough" means that the distance between two frequencies is larger than $2 v_{0}$. In that case, the corresponding term (frequency, phase, and amplitude) is removed from the signal, and we apply the procedure again to find the next term. If, in one step of the procedure, we find a frequency separated from a previously determined one by less than $2 v_{0}$, we put this term back in the signal to determine it again. Sometimes it is enough, and the procedure continues with the discovery of new frequencies. But, if the problem occurs again with the same term, then we determine all the previous terms again, one by one. This method was firstly used by Champenois (1999). He shows that, in some conditions, the iterative procedure eliminates the problem of the influence of one frequency on the others.

\section{Synthetic analysis}

In celestial mechanics, it is usually convenient to split the orbital spectrum in two parts: the short period terms (typically a few periods) and the long period terms (few pericenter and node periods). As discussed in Sect. 2, the Galilean system spectrum has short periods that are less than a day and long periods of the order of a few centuries. So, for the practical use of the frequency analysis, it is better to cut the initial signal with respect to these two parts. This method avoids manipulating a file of several hundreds of megabytes: the time span $T$ must be long enough to determine all the long period terms, and the step size of the signal must be small enough to determine the shortest period. We then have to use digital filtering to obtain two signals for which the sum of both is the original one. Indeed, the signal corresponding to the short period terms can be set to a much smaller time span than the original signal although the same step size can be used. The signal corresponding to the long period terms can be set with a much larger step size than the original signal, but with the same time span.

\subsection{Digital filtering}

The digital filtering used here is very similar to the one used in Duriez \& Vienne (1997). The reader can find the details of the procedure in that paper. In particular, we used a two-step filtering process to avoid the use of a single filter with too high of 
Table 1. Characteristics of the two-step filtering used to remove the short period terms from the signal in the Galilean dynamical system.

\begin{tabular}{ccc}
\hline \hline & Filter 1 & Filter 2 \\
\hline$\Delta t$ (days) & 0.24 & 3.36 \\
$T_{0}$ (days) & 6.72 & 60 \\
$T_{1}$ (days) & 140 & 140 \\
$\delta t$ (days) & 3.36 & 26.88 \\
$2 M+1$ & 231 & 247 \\
$\rho$ & $10^{-7}$ & $10^{-7}$ \\
$\alpha$ & $10^{-6}$ & $10^{-6}$ \\
$\Delta f$ & 0.034 & 0.032 \\
\hline
\end{tabular}

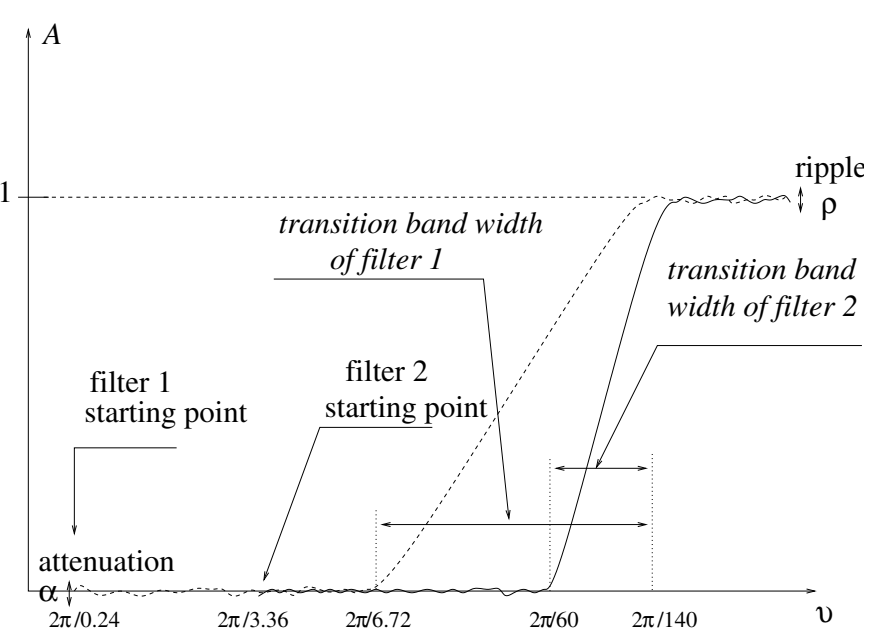

Fig. 1. The characteristics of the two-step filtering.

a number of coefficients. Table 1 summarises the characteristics of the two filters we used. Figure 1 visualises the characteristics of the two-step filtering. $\Delta t$ denotes the step size in input for filtering, while $\delta t$ is the output step size; $\left[T_{0}, T_{1}\right]$ and $\Delta f$ are the cutting intervals expressed, respectively, in days and in sampling frequency; $2 M+1$ is the filter length (odd number); and $\rho$ and $\alpha$ denote the pass-band ripple and the stop-band attenuation.

This two-step filtering method was applied at the output of our numerical integration, after converting Cartesian coordinates into Keplerian elements. To filter the mean longitude signal, we first subtracted a mean frequency empirically, and then refined it with a least squares fit. To obtain all long periodic terms, we simulated the Galilean satellites' motion over $+/-850$ years, starting at the Julian epoch 2433282.5 (January 1st, 1950, at 0h). The numerical accuracy of such integration was estimated to a few hundreds of meters. After filtering, our sample covered 1700 years with a step size of 26.88 days. This span is long enough to detect the longest periodic term of the Galilean system equal to 562 years (node of Callisto).

\subsection{Long period treatment}

To analyse the long period terms of the Galilean system, we used the frequency analysis method presented in Sect. 4 on the filtered signal. The efficiency of the quasi-periodic representation was estimated by comparing the initial signal and the reconstructed one. We obtained good results for all elements (agreement around few kilometers), except for the satellite mean longitudes (a few tens of kilometers). Problems arise because of the presence of very long solar periodic terms that cannot be analysed with only a 1700 year time span. These terms appear in all variables, except the semi-major axis. The recognition of such terms would have needed a much longer time span, about a few hundred thousand years, because of the period of revolution of the perihelies and nodes of the planets around the Sun. With a step size as short as a day, this would also increase the numerical error in the integration process too much. Of course, these very long period terms do not appear to be periodic over 1700 years only. To overcome this problem, in all variables except the semi-major axis, we fit the residuals polluted by the very long period terms with Tchebychev polynomials. An optimal method was found in four steps. First, we filtered the residuals to remove undetected short period terms and kept only the very long period part. Then we fit the signal with low-order Tchebychev polynomials (orders less than 8). Such polynomials were then subtracted from the initial residuals, and we could start our frequency analysis treatment again. In practice, few iterations were enough to have a precision of a few tens of kilometers. Figure 2 presents all four steps of this method applied to the mean longitude of Callisto. To preserve space in this paper, these Tchebychev polynomials are not given here, but are available upon request to the authors. The long period terms found by frequency analysis in the mean longitudes (after subtracting the Tchebychev polynomials) have amplitudes greater than $2.2 \mathrm{~km}$, $6.2 \mathrm{~km}, 6.1 \mathrm{~km}$, and $21.8 \mathrm{~km}$ (respectively, in the mean longitudes of Io, Europa, Ganymede, and Callisto). This truncation level explains the precision of a few tens of kilometers that has been obtained. In other variables, we were able to obtain truncation levels half of those for mean longitudes.

\subsection{Short period treatment}

The short period spectrum is analysed by subtracting the recognized long period terms on an unfiltered sample produced by our numerical integration. We can use a much less extended time span since long periodic terms are removed. A step size of 0.24 day was used and allowed us to detect all the short period terms longer than 0.48 day. A time span of 80 years was required to separate the close frequencies of the system. We were able to adopt a truncation level of the short period terms that was less than that of long period ones (a level as low as about $0.2 \mathrm{~km}$ ). Then, some residual long period terms were still able to be detected in the signal (at a level less than the amplitudes cited above in the long period treatment). We again used the filtering process with Tchebychev polynomials to progress in frequency analysis up to this truncation level. However, the long period terms found in this way, along with the short period ones, seem to be less reliable when trying to reconstruct their frequencies as combinations of the fundamental frequencies (see Sect. 6).

\subsection{Precision}

The trigonometric series and Tchebychev polynomials obtained by the above method represent what are called "L1 ephemerides". The corresponding tables are available by $\mathrm{ftp}^{2}$, as well as by a FORTRAN subroutine that can be used to compute ephemerides of the Galilean satellites. The subroutine output was compared to an unfiltered sampling delivered by our numerical model. The final accuracy (internal precision) over one century (centred on initial epoch 2433282.5 ) of our frequency representation was found to be of a few kilometers for Io, 20 kilometers for Europa and Ganymede, and 35 kilometers for Callisto. Over 1700 years (again centred on a J1950.0 epoch),

\footnotetext{
${ }^{2}$ ftp://ftp.imcce.fr/pub/ephem/satel/galilean/L1
} 

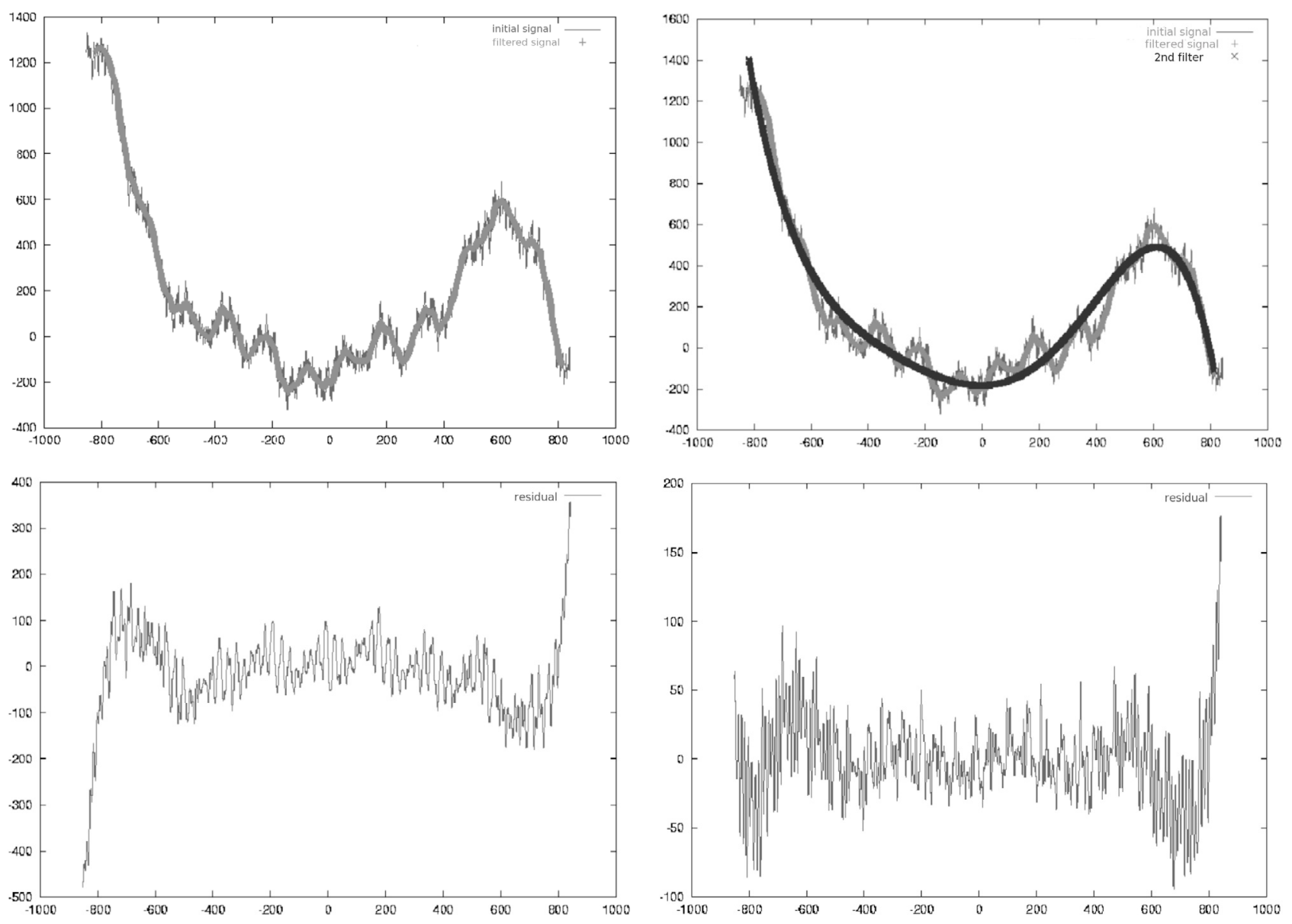

Fig. 2. Four step method used to reconstruct Callisto's mean longitude. $X$-axes are in years, and $Y$-axes are in kilometers. Figures on top present the residuals before the Tchebychev treatment. On the left, a filtering process is performed to remove short period terms. On the right, a Tchebychev polynomial is fit on the filtered signal. Figures on the bottom present the residuals after subtracting the Tchebychev polynomial (left) and applying the frequency analysis treatment again (right).

our representation is accurate to 25 kilometers for Io, 90 kilometers for Europa and Ganymede, and 150 kilometers for Callisto. Such accuracy is just the internal precision of our representation. The accuracy of L1 ephemerides (external precision) also introduces the error coming from the fit process (mainly due to observational errors). Considering this, the global accuracy of L1 ephemerides can be estimated to 25 kilometers for Io, 40 kilometers for Europa and Ganymede, and 55 kilometers for Callisto over one century.

\section{Identification and synthetic representation}

We first identified the fundamental arguments in the numerical representations issued from the frequency analysis process. These arguments are:

$L_{i}$ : the linear part in the mean longitudes of the satellite $i$.

$\varpi_{i}$ : the four proper modes in the longitudes of the pericenters. $\Omega_{i}$ : the four proper modes in the longitudes of the nodes.

$\Omega_{0}$ : the proper mode related to the longitude of the node of the Laplacian plane.

$v$ : the great inequality $L_{1}-2 L_{2}$ or $L_{2}-2 L_{3}+180^{\circ}$.

We remind the reader that $\left(L_{1}-2 L_{2}\right)-\left(L_{2}-2 L_{3}\right)=L_{1}-$ $3 L_{2}+2 L_{3}$ oscillates around $180^{\circ}$. This libration is the main effect of the Laplacian resonance.

$\Psi$ : the argument of the Laplacian resonance. $\rho$ : the De Haerdtl great inequality $3 L_{3}-7 L_{4}$.

$L_{\mathrm{S}}$ : the linear part, in time, in the mean longitude of the Sun.

Except $v$ and $\rho$, which are connected to some $L_{i}$, these arguments represent the minimum set able to represent the full dynamics of the Galilean satellites.

Table 2 gives the values of these arguments and the solutions from which they issue. These solutions are given in Tables 3 to 18 , where each term is in the form of a signed numerical coefficient that is supposed to be in the factor of a trigonometric function (sine, cosine, or complex exponential) whose argument corresponds to the formulae:

phase + frequency $\times T$,

where $T=\mathrm{DJ}-2433282.5$ (that is, the time from J1950, in days). The time scale is TDB. The short period terms are presented together with the long period ones, simply by decreasing amplitudes. These amplitudes are expressed in kilometers, by a simple conversion of $\mathrm{AU}$ in $\mathrm{km}$ in the semi-major axis, or by multiplying radians (in other variables) by the constant part of the semi-major axis (in $\mathrm{km}$ ) of the corresponding satellite. The solutions are expressed in cosine for the semi-major axis $a_{i}$, in sine for the mean longitudes $\mathcal{L}_{i}$, and in complex exponential for the variables $z_{i}$ (eccentricity-pericenter) and $\zeta_{i}$ (inclinationnode).

The recognition of the fundamental arguments in these solutions was possible because previous analytical works, such as 
Table 2. Fundamental arguments of the Galilean system used for the identification. Arguments are in the form Phase + Frequency $\times T$ with $T=\mathrm{JD}-2433282.5$. The argument $v$ is also very close to the combinations $L_{1}-2 L_{2}$ and $L_{2}-2 L_{3}+180^{\circ}$.

\begin{tabular}{crrrrc}
\hline \hline $\begin{array}{c}\text { Fundamental } \\
\text { argument }\end{array}$ & $\begin{array}{c}\text { Frequency } \\
\text { (rad/day) }\end{array}$ & \multicolumn{1}{c}{$\begin{array}{c}\text { Phase } \\
(\mathrm{deg})\end{array}$} & $\begin{array}{c}\text { Period } \\
\text { (day) }\end{array}$ & $\begin{array}{r}\text { Period } \\
\text { (year) }\end{array}$ & $\begin{array}{c}\text { Issued from } \\
\text { solution }\end{array}$ \\
\hline$L_{1}$ & 3.551552286182 & 82.861918 & 1.769 & 0.005 & $\mathcal{L}_{1}$ \\
$L_{2}$ & 1.769322711123 & 338.598517 & 3.551 & 0.010 & $\mathcal{L}_{2}$ \\
$L_{3}$ & 0.878207923589 & 16.467319 & 7.154 & 0.019 & $\mathcal{L}_{3}$ \\
$L_{4}$ & 0.376486233434 & 339.256972 & 16.689 & 0.046 & $\mathcal{L}_{4}$ \\
$L_{\mathrm{S}}$ & 0.001450183749 & 318.603037 & 4332.938 & 11.863 & $z_{4}$ \\
$\Psi$ & 0.003050648672 & 109.319546 & 2059.622 & 5.639 & $\mathcal{L}_{2}$ \\
$\nu$ & 0.012906864147 & 125.663720 & 486.809 & 1.333 & $z_{1}, z_{2}$ or $z_{3}$ \\
$\rho$ & -0.000779862000 & 194.603151 & 8056.791 & 22.058 & $=3 L_{3}-7 L_{4}$ \\
$\varpi_{1}$ & 0.002664253355 & 25.288760 & 2358.328 & 6.457 & $z_{1}$ \\
$\varpi_{2}$ & 0.000677973430 & 157.191935 & 9267.598 & 25.373 & $z_{2}$ \\
$\varpi_{3}$ & 0.000127274130 & 121.789605 & 49367.340 & 135.160 & $z_{3}$ \\
$\varpi_{4}$ & 0.000032065099 & 319.917125 & 195950.909 & 536.484 & $z_{4}$ \\
$\Omega_{1}$ & -0.002315096098 & 160.223177 & 2714.006 & 7.431 & $\zeta_{1}$ \\
$\Omega_{2}$ & -0.000569206405 & 60.029150 & 11038.500 & 30.222 & $\zeta_{2}$ \\
$\Omega_{3}$ & -0.000124913071 & 191.189501 & 50300.463 & 137.715 & $\zeta_{3}$ \\
$\Omega_{4}$ & -0.000030561255 & 342.180346 & 205593.170 & 562.883 & $\zeta_{4}$ \\
$\Omega_{0}$ & 0.000000000000 & 138.277188 & $\infty$ & $\infty$ & $\zeta_{4}$ \\
\hline
\end{tabular}

Table 3. Solution for the variable $a_{1}$ (semi-major axis of Io). The series is expressed in cosine.

\begin{tabular}{rrccrc}
\hline \hline $\begin{array}{r}\text { Amplitude } \\
(\mathrm{km})\end{array}$ & $\begin{array}{c}\text { Phase } \\
(\mathrm{deg})\end{array}$ & $\begin{array}{c}\text { Frequency } \\
(\mathrm{rad} / \text { day })\end{array}$ & Identification & $\begin{array}{r}\text { Freq. diff. } \\
(\mathrm{rad} / \mathrm{day})\end{array}$ & $\begin{array}{r}\text { Ph. diff. } \\
(\mathrm{deg})\end{array}$ \\
\hline 422029.958 & 0.00000 & 0.0000000000 & & & \\
11.400 & 208.51597 & 3.5644591656 & $2 L_{1}-2 L_{2}$ & $-0.155 \mathrm{D}-07$ & 0.01083 \\
2.706 & 57.04065 & 7.1289183312 & $4 L_{1}-4 L_{2}$ & $-0.310 \mathrm{D}-07$ & 0.01295 \\
2.578 & 104.25820 & 1.7822295778 & $L_{1}-L_{2}$ & $-0.270 \mathrm{D}-08$ & 0.00520 \\
1.522 & 161.29083 & 8.9111478635 & $5 L_{1}-5 L_{2}$ & $0.118 \mathrm{D}-07$ & 0.02618 \\
1.418 & 199.16142 & 8.0200331113 & $3 L_{1}-3 L_{3}$ & $-0.235 \mathrm{D}-07$ & 0.02238 \\
1.379 & 265.54878 & 10.6933774362 & $4 L_{1}-4 L_{3}$ & $0.142 \mathrm{D}-07$ & 0.02962 \\
\hline
\end{tabular}

that of Ferraz-Mello (1979) or of Duriez (1982) give, at least, their period. It appears that the same fundamental argument is present in several solutions, generally with very small differences (for instance $\varpi_{3}$, in solutions $z_{1}, z_{2}, z_{3}$, or $z_{4}$ ). We chose the argument in one of these solutions that generally correspond to the term whose amplitude is the largest, to have the best determination of the phase (for instance $\varpi_{3}$ was issued from solution $z_{3}$ ).

After that, we tried to identify each frequency in the solutions as integer combinations of the fundamental frequencies. The redundancies of $v$ and $\rho$ with the relating combinations of $L_{i}$ where used to identify the frequencies in the long period part of the solutions more easily.

In most cases, the frequency of each term is recognized as an integer combination of the frequencies of the fundamental arguments, by trying all possible combinations with increasing integers until the best agreement is reached, but also by respecting d'Alembert's rule (except for the solar terms, see below). As a consequence, higher integers for the arguments $\varpi$ and $\Omega$ corresponds to higher degree in $z$ and $\zeta$ (that is in eccentricities and inclinations). We then verify that the same combination of integer applied on the phases of the fundamental arguments allow us to recover the phase of the term (a difference of $180^{\circ}$ was converted by changing the sign of the amplitude). Tables 3 to 18 show how each term is identified as an integer combination of the fundamental arguments. In the two last columns, we also give the difference in frequency and phase between their numerical values issued from the frequency analysis process and their evaluation by the related combination of fundamental arguments. Identification is best when the differences are smallest.
The solar terms are those depending on the argument $L_{\mathrm{S}}$, and, to less of an extent, those with the argument $\Omega_{0}$. Generally, these terms also depend on the eccentricity and/or inclination of solar Jovicentric motion, hence on the very long period associated with the motions of the pericenter and/or node of the solar orbit. The time scale of the corresponding motion is much longer than that of the satellite. Thus, the solar frequencies of the pericenter and the node cannot be separated in our analysis. As a consequence, we can recognize a frequency close to its mean longitude frequency, but not the phase. This is why the values in column "phase difference" are not significant for solar terms and hence not given. Some other terms (essentially small terms) are not well identified; these are considered as doubtful and indicated by a star symbol (*) in the tables. These doubtful terms come essentially from the long period part of the solution found in the short period treatment (Sect. 5.3). The given identification is considered doubtful when it corresponds to a very improbable inequality (like $2 L_{\mathrm{S}}+v-\varpi_{3}-\Omega_{3}$ in solution $z_{2}$ or like $2 L_{S}-\Psi+\varpi_{1}-\Omega_{4}$ in the mean longitude of Io) or when the difference in frequency is larger than about $10^{-5} \mathrm{rad} /$ day. For these terms and for the solar terms, it is better to use the numerical value of the frequency and the phase to evaluate the term instead of its doubtful identification.

In Tables 3 to 18, we have used a truncation level of 1 kilometer. There are some more terms with lower amplitudes, but they are not really significant from an analytical point of view. We recall that the internal precision of L1 is a few hundred of meters. However, we give some smaller terms that are identified, for example, as being related to the De Haerdtl great inequality or to a new, potentially interesting inequality. These 
terms concern an inequality between the three last satellites: $L_{2}-3 L_{3}+2 L_{4}{ }^{3}$. This inequality of a second order of masses appears in the solution of the semi-major axis and the mean longitude of Ganymede. The corresponding amplitudes are very small (respectively, 0.36 and 4.53 kilometers), and the period is 66 days. So, it is not so far from a resonance. Can this inequality have had an influence upon the evolution of the dynamical system in the past?

More generally, thanks to the identification performed here, this synthetic representation holds some analytical information, in the sense that each term may represent a specific perturbation.

The Galilean system is sometimes referted to with two more resonances involving the arguments $L_{1}-2 L_{2}+\varpi_{1}$ and $L_{2}-2 L_{3}+\varpi_{2}$. The reason is that there are librations of these arguments. However, this condition is not sufficient. If it were, such resonances should have brought two new fundamental frequencies (libration frequencies) to the system. Nonetheless, we succeeded in recognizing all frequencies as a combination of the fundamental arguments shown in Table 2. Hence, we cannot confirm the existence of such resonances.

\section{Conclusion}

We have given the trigonometric series whose arguments are integer combinations of the fundamental arguments of the dynamical system, which are also given. According to the Sampson-Lieske theory, a new representation of the Galilean orbital motions deduced from recent ephemerides is now available. It is a useful base for other dynamical studies on these satellites: the understanding, in the perturbation sense, of the orbital motions, and then the history and evolution of the dynamical system under tidal effects, as well as the rotation of the bodies. However, the doubtful identification of some terms may still be corrected. Our work to improve the frequency analysis process, to obtain better accuracy in the determination of the frequencies, is still in progress.
The present solutions benefited from L1 ephemerides, which is a former fit to the observations, recently performed in Lainey et al. (2004b). The tool is now complete and can be easily updated in light of new observations, such as spatial ones (Galileo spacecraft) or Earth-based ones (mutual events campaigns).

A FORTRAN subroutine computing L1 ephemerides by means of the present synthetic representation is available on request.

Acknowledgements. This work benefited from the support of the European Community's Improving Human Potential Programme under contract RTN2-2001-00414, MAGE.

\section{References}

Champenois, S. 1999, thesis, Observatoire de Paris

Champenois, S., \& Vienne, A. 1999a, Celes. Mech. Dyn. Astr., 74, 111

Champenois, S., \& Vienne, A. 1999b, Icarus, 140, 106

Duriez, L. 1982, Celes. Mech. Dyn. Astr., 26, 31

Duriez, L., \& Vienne, A. 1997, A\&A, 324, 366

Duriez, L. 1990, Le Développement de la Fonction Perturbatrice. In Modern methods in Celestial Mechanics, ed. D. Benest, \& C. Froeschlé (Éditions Frontières), 35

Duriez, L. 2003, Maple routines for computing disturbing functions, http://www. univ-lille1.fr/lal/mecanique_celeste.html

Ferraz-Mello, S. 1979, Dynamics of the Galilean satellites, ed. Universidade de Sao Paulo

Henrard, J. 2005a, Celes. Mech. Dyn. Astr., 91, 131

Henrard, J. 2005b, Icarus, 178, 144

Lainey, V. 2002, thesis, Observatoire de Paris

Lainey, V., Duriez, L., \& Vienne, A. 2004a, A\&A, 420, 1171

Lainey, V., Arlot, J. E., \& Vienne, A. 2004b, A\&A, 427, 371

Laskar, J. 1993, Physica D, 67, 257

Lieske, J. H. 1977, A\&A, 56, 333

Noyelles, B. 2005, thesis, Observatoire de Paris

Sagnier, J. L. 1975, Celes. Mech. Dyn. Astr., 12, 19

Sampson, R. A. 1921, Mem. R. Astron. Soc., 63

Simon, J.-L., Bretagnon, P., Chapront, J., et al. 1994, A\&A, 282, 663

Thuillot, W. 1984, Celes. Mech. Dyn. Astr., 34, 245

Vienne, A., \& Duriez, L. 1992, A\&A, 257, 331

Vienne, A., \& Duriez, L. 1995, A\&A, 297, 588

Vu, D. T. 1982, Celes. Mech. Dyn. Astr., 26, 265

${ }^{3}$ Not to be confused with the Laplacian resonance, which involves the three first satellites: $L_{1}-3 L_{2}+2 L_{3}$. 
V. Lainey et al.: Synthetic representation of the Galilean satellites' orbital motions, Online Material p 1

\section{Online Material}


V. Lainey et al.: Synthetic representation of the Galilean satellites' orbital motions, Online Material p 2

Table 4. Solution for $\mathcal{L}_{1}$ (mean longitude of Io): $\mathcal{L}_{1}=L_{1}+$ series expressed in sine, with $L_{1}=1.4462132960212235+3.551552286182 \times T$.

\begin{tabular}{|c|c|c|c|c|c|c|}
\hline $\begin{array}{r}\text { Amplitude } \\
(\mathrm{km})\end{array}$ & $\begin{array}{r}\text { Phase } \\
\text { (deg) }\end{array}$ & $\begin{array}{r}\text { Frequency } \\
\text { (rad/day) }\end{array}$ & Identification & $\begin{array}{r}\text { Freq. diff. } \\
\text { (rad/day) }\end{array}$ & $\begin{array}{r}\text { Ph. diff. } \\
\text { (deg) }\end{array}$ & \\
\hline-81.252 & 282.86691 & 0.0135848366 & $v+\varpi_{2}$ & $0.994 \mathrm{D}-09$ & -0.01125 & \\
\hline-40.971 & 247.45358 & 0.0130341384 & $v+\varpi_{3}$ & $-0.155 \mathrm{D}-09$ & -0.00026 & \\
\hline-37.933 & 109.32044 & 0.0030506487 & $\Psi$ & $0.363 \mathrm{D}-06$ & -0.00084 & \\
\hline-23.343 & 85.57787 & 0.0129389289 & $v+\varpi_{4}$ & $0.334 \mathrm{D}-09$ & 0.00298 & \\
\hline-21.253 & 208.61506 & 3.5644591050 & $2 L_{1}-2 L_{2}$ & $0.452 \mathrm{D}-07$ & -0.08826 & \\
\hline-18.756 & 104.25814 & 1.7822295778 & $L_{1}-L_{2}$ & $-0.270 \mathrm{D}-08$ & 0.00526 & \\
\hline 17.644 & 150.95338 & 0.0155711172 & $v+\varpi_{1}$ & $0.280 \mathrm{D}-09$ & -0.00090 & \\
\hline 15.715 & 122.62695 & 0.0014500977 & $L_{\mathrm{S}}$ & $0.860 \mathrm{D}-07$ & & \\
\hline-9.894 & 11.38579 & 0.0246753155 & $2 v+2 \Omega_{2}$ & $-0.249 \mathrm{D}-10$ & -0.00005 & \\
\hline-6.766 & 161.59399 & 0.0000951962 & $\varpi_{3}-\varpi_{4}$ & $0.128 \mathrm{D}-07$ & 0.27849 & \\
\hline-5.024 & 57.02165 & 7.1289183312 & $4 L_{1}-4 L_{2}$ & $-0.310 \mathrm{D}-07$ & 0.03195 & \\
\hline-4.601 & 66.38715 & 2.6733443704 & $L_{1}-L_{3}$ & $-0.783 \mathrm{D}-08$ & 0.00745 & \\
\hline 3.681 & 131.75213 & 0.0004445681 & $-\Omega_{2}+\Omega_{3}$ & $-0.275 \mathrm{D}-06$ & -0.59178 & \\
\hline 3.470 & 48.54310 & 0.0005498008 & $\varpi_{2}-\varpi_{4}+\Omega_{3}-\Omega_{4}$ & $0.176 \mathrm{D}-05$ & -2.25913 & \\
\hline 3.181 & 175.58064 & 0.0006482675 & $\varpi_{2}-\varpi_{3}-\Omega_{3}+\Omega_{4}$ & $-0.322 \mathrm{D}-05$ & 10.81254 & \\
\hline-2.593 & 161.29048 & 8.9111478635 & $5 L_{1}-5 L_{2}$ & $0.118 \mathrm{D}-07$ & 0.02653 & \\
\hline-2.430 & 199.16149 & 8.0200331113 & $3 L_{1}-3 L_{3}$ & $-0.235 \mathrm{D}-07$ & 0.02231 & \\
\hline-2.245 & 85.66898 & 0.0029001291 & $2 L_{\mathrm{S}}$ & $0.238 \mathrm{D}-06$ & & \\
\hline-2.160 & 265.54919 & 10.6933774362 & $4 L_{1}-4 L_{3}$ & $0.142 \mathrm{D}-07$ & 0.02921 & \\
\hline-2.018 & 282.10444 & 0.0030554834 & $2 L_{\mathrm{S}}-\Omega_{3}-\Omega_{4}$ & $0.358 \mathrm{D}-06$ & & * \\
\hline 1.923 & 112.21434 & 0.0229286259 & $2 v+\Omega_{1}+\Omega_{2}$ & $0.800 \mathrm{D}-06$ & -0.63457 & \\
\hline 1.823 & 90.77307 & 0.0156771125 & $2 L_{\mathrm{S}}+v-\varpi_{3}$ & $0.285 \mathrm{D}-05$ & & * \\
\hline 1.590 & 132.77525 & 5.3466887181 & $2 L_{1}-2 L_{3}$ & $0.708 \mathrm{D}-08$ & 0.01395 & \\
\hline-1.325 & 127.10539 & 0.0251554892 & $-L_{\mathrm{S}}+2 v+\varpi_{2}-\Omega_{4}$ & $-0.834 \mathrm{D}-04$ & -19.36940 & * \\
\hline 1.027 & 48.88513 & 0.0025426969 & $2 L_{\mathrm{S}}-\Psi+\varpi_{1}-\Omega_{4}$ & $0.147 \mathrm{D}-05$ & & $*$ \\
\hline
\end{tabular}

Table 5. Solution for the variable $z_{1}$ (eccentricity and pericenter of Io). The series is expressed in complex exponentials.

\begin{tabular}{rrccrr}
\hline \hline $\begin{array}{r}\text { Amplitude } \\
(\mathrm{km})\end{array}$ & $\begin{array}{c}\text { Phase } \\
(\mathrm{deg})\end{array}$ & $\begin{array}{c}\text { Frequency } \\
(\mathrm{rad} / \mathrm{day})\end{array}$ & Identification & $\begin{array}{r}\text { Freq. diff. } \\
\text { (rad/day) }\end{array}$ & $\begin{array}{r}\text { Ph. diff. } \\
(\mathrm{deg})\end{array}$ \\
\hline 1751.882 & 234.33628 & -0.0129068641 & $-v$ & $0.000 \mathrm{D}+00$ & 0.00000 \\
264.213 & 82.86052 & 3.5515522950 & $L_{1}$ & $-0.880 \mathrm{D}-08$ & 0.00140 \\
14.887 & 121.78955 & 0.0001272742 & $\varpi_{3}$ & $-0.354 \mathrm{D}-10$ & 0.00005 \\
8.364 & 319.91454 & 0.0000320658 & $\varpi_{4}$ & $-0.652 \mathrm{D}-09$ & 0.00258 \\
6.179 & 25.28876 & 0.0026642534 & $\varpi_{1}$ & $0.000 \mathrm{D}+00$ & 0.00000 \\
4.168 & 25.82671 & -3.5773660260 & $-3 L_{1}+4 L_{2}$ & $0.119 \mathrm{D}-07$ & -0.01840 \\
-4.086 & 338.60240 & 1.7693227079 & $L_{2}$ & $0.318 \mathrm{D}-08$ & -0.00388 \\
-3.506 & 16.47338 & 0.8782079195 & $L_{3}$ & $0.407 \mathrm{D}-08$ & -0.00606 \\
2.519 & 290.72310 & 7.1160118049 & $3 L_{1}-2 L_{2}$ & $-0.369 \mathrm{D}-06$ & 0.66562 \\
-2.204 & 157.33840 & 0.0006779610 & $\varpi_{2}$ & $0.124 \mathrm{D}-07$ & -0.14646 \\
1.964 & 281.56981 & -5.3595956184 & $-4 L_{1}+5 L_{2}$ & $0.293 \mathrm{D}-07$ & -0.02490 \\
1.939 & 243.69908 & -4.4684808201 & $-2 L_{1}+3 L_{3}$ & $0.185 \mathrm{D}-07$ & -0.02096 \\
-1.592 & 310.08529 & -1.7951364446 & $-L_{1}+2 L_{3}$ & $0.558 \mathrm{D}-08$ & -0.01257 \\
1.579 & 177.31174 & -7.1418251641 & $-3 L_{1}+4 L_{3}$ & $-0.983 \mathrm{D}-10$ & -0.02822 \\
\hline
\end{tabular}

Table 6. Solution for the variable $\zeta_{1}$ (inclination and node of Io). The series is expressed in complex exponentials.

\begin{tabular}{rrccrr}
\hline \hline $\begin{array}{r}\text { Amplitude } \\
(\mathrm{km})\end{array}$ & $\begin{array}{c}\text { Phase } \\
(\mathrm{deg})\end{array}$ & $\begin{array}{c}\text { Frequency } \\
(\mathrm{rad} / \mathrm{day})\end{array}$ & Identification & $\begin{array}{r}\text { Freq. diff. } \\
(\mathrm{rad} / \mathrm{day})\end{array}$ & $\begin{array}{r}\text { Ph. diff. } \\
(\mathrm{deg})\end{array}$ \\
\hline 132.609 & 160.22318 & -0.0023150961 & $\Omega_{1}$ & $0.000 \mathrm{D}+00$ & 0.00000 \\
38.159 & 60.02914 & -0.0005692064 & $\Omega_{2}$ & $-0.234 \mathrm{D}-10$ & 0.00001 \\
7.145 & 138.37395 & 0.0000000000 & $\Omega_{0}$ & $0.000 \mathrm{D}+00$ & -0.09677 \\
6.940 & 191.18949 & -0.0001249131 & $\Omega_{3}$ & $0.139 \mathrm{D}-11$ & 0.00001 \\
2.339 & 342.17155 & -0.0000305612 & $\Omega_{4}$ & $-0.905 \mathrm{D}-10$ & 0.00880 \\
1.513 & 48.64339 & -0.0252445219 & $-2 v-\Omega_{2}$ & $0.127 \mathrm{D}-10$ & 0.00002 \\
1.021 & 318.58614 & 0.0029003681 & $2 L_{\mathrm{S}}$ & $-0.646 \mathrm{D}-09$ & \\
\hline
\end{tabular}


V. Lainey et al.: Synthetic representation of the Galilean satellites' orbital motions, Online Material p 3

Table 7. Solution for the variable $a_{2}$ (semi-major axis of Europa). The series is expressed in cosine.

\begin{tabular}{rrrcrr}
\hline \hline $\begin{array}{r}\text { Amplitude } \\
(\mathrm{km})\end{array}$ & $\begin{array}{c}\text { Phase } \\
(\mathrm{deg})\end{array}$ & $\begin{array}{c}\text { Frequency } \\
(\mathrm{rad} / \mathrm{day})\end{array}$ & Identification & $\begin{array}{r}\text { Freq. diff. } \\
(\mathrm{rad} / \mathrm{day})\end{array}$ & $\begin{array}{r}\text { Ph. diff. } \\
(\mathrm{deg})\end{array}$ \\
\hline 671261.171 & 0.00000 & 0.0000000000 & & & \\
64.692 & 104.25801 & 1.7822295778 & $L_{1}-L_{2}$ & $-0.270 \mathrm{D}-08$ & 0.00539 \\
23.990 & 246.38747 & 2.6733443704 & $3 L_{2}-3 L_{3}$ & $-0.782 \mathrm{D}-08$ & 0.00612 \\
-15.246 & 312.77469 & 5.3466887181 & $3 L_{1}-3 L_{2}$ & $0.707 \mathrm{D}-08$ & 0.01551 \\
13.834 & 322.12913 & 0.8911147889 & $L_{2}-L_{3}$ & $-0.134 \mathrm{D}-08$ & 0.00207 \\
-7.834 & 208.51565 & 3.5644591656 & $2 L_{1}-2 L_{2}$ & $-0.155 \mathrm{D}-07$ & 0.01115 \\
7.652 & 170.64578 & 4.4555739318 & $5 L_{2}-5 L_{3}$ & $0.592 \mathrm{D}-08$ & 0.01021 \\
-4.666 & 56.99014 & 7.1289183312 & $4 L_{1}-4 L_{2}$ & $-0.310 \mathrm{D}-07$ & 0.06346 \\
-4.082 & 161.25599 & 8.9111478635 & $5 L_{1}-5 L_{2}$ & $0.118 \mathrm{D}-07$ & 0.06102 \\
3.474 & 358.71991 & 2.7856729212 & $2 L_{2}-2 L_{4}$ & $0.342 \mathrm{D}-07$ & -0.03682 \\
-2.712 & 247.45298 & 0.0130341383 & $v+\varpi_{3}$ & $-0.319 \mathrm{D}-10$ & 0.00034 \\
2.617 & 94.90439 & 6.2378035398 & $7 L_{2}-7 L_{3}$ & $-0.271 \mathrm{D}-07$ & 0.01399 \\
-1.838 & 265.97440 & 10.6933772542 & $6 L_{1}-6 L_{2}$ & $0.196 \mathrm{D}-06$ & -0.39399 \\
-1.427 & 85.58005 & 0.0129389288 & $v+\varpi_{4}$ & $0.425 \mathrm{D}-09$ & 0.00080 \\
-1.270 & 9.82442 & 12.4756070797 & $7 L_{1}-7 L_{2}$ & $-0.543 \mathrm{D}-07$ & 0.01939 \\
1.076 & 282.90788 & 0.0135848330 & $v+\varpi_{2}$ & $0.456 \mathrm{D}-08$ & -0.05222 \\
1.034 & 358.03414 & 4.1785094280 & $3 L_{2}-3 L_{4}$ & $0.502 \mathrm{D}-08$ & -0.00951 \\
\hline
\end{tabular}

Table 8. Solution for $\mathcal{L}_{2}$ (mean longitude of Europa): $\mathcal{L}_{2}=L_{2}+$ series expressed in sine, with $L_{2}=0.3735263437471362+1.769322711123 \times T$.

\begin{tabular}{|c|c|c|c|c|c|c|}
\hline $\begin{array}{r}\text { Amplitude } \\
(\mathrm{km})\end{array}$ & $\begin{array}{r}\text { Phase } \\
\text { (deg) }\end{array}$ & $\begin{array}{r}\text { Frequency } \\
(\mathrm{rad} / \text { day })\end{array}$ & Identification & $\begin{array}{r}\text { Freq. diff. } \\
\text { (rad/day) }\end{array}$ & $\begin{array}{r}\text { Ph. diff. } \\
\text { (deg) }\end{array}$ & \\
\hline 575.703 & 247.45298 & 0.0130341383 & $v+\varpi_{3}$ & $-0.311 \mathrm{D}-10$ & 0.00034 & \\
\hline 305.396 & 85.58002 & 0.0129389288 & $v+\varpi_{4}$ & 0.426D-09 & 0.00083 & \\
\hline 218.089 & 104.25823 & 1.7822295778 & $L_{1}-L_{2}$ & $-0.270 \mathrm{D}-08$ & 0.00517 & \\
\hline-206.362 & 282.90958 & 0.0135848329 & $v+\varpi_{2}$ & $0.471 \mathrm{D}-08$ & -0.05392 & \\
\hline 133.070 & 109.31960 & 0.0030510121 & $\Psi$ & $0.000 \mathrm{D}+00$ & 0.00000 & \\
\hline 123.114 & 122.62932 & 0.0014500979 & $L_{\mathrm{S}}$ & 0.859D-07 & & \\
\hline-96.285 & 322.12914 & 0.8911147889 & $L_{2}-L_{3}$ & $-0.134 \mathrm{D}-08$ & 0.00206 & \\
\hline-51.817 & 246.38746 & 2.6733443704 & $3 L_{2}-3 L_{3}$ & $-0.782 \mathrm{D}-08$ & 0.00613 & \\
\hline-42.443 & 150.95371 & 0.0155711171 & $v+\varpi_{1}$ & 0.417D-09 & -0.00123 & \\
\hline 29.990 & 312.77450 & 5.3466887181 & $3 L_{1}-3 L_{2}$ & $0.707 \mathrm{D}-08$ & 0.01570 & \\
\hline 29.306 & 208.51601 & 3.5644591656 & $2 L_{1}-2 L_{2}$ & $-0.155 \mathrm{D}-07$ & 0.01079 & \\
\hline 23.439 & 162.08900 & 0.0000298857 & $-\Omega_{4}+\Omega_{0}$ & $0.676 \mathrm{D}-06$ & -5.99217 & \\
\hline-21.864 & 307.80100 & 0.0001249523 & $-\Omega_{3}+\Omega_{0}$ & $-0.393 \mathrm{D}-07$ & -0.71332 & \\
\hline 13.816 & 87.42456 & 0.0029001316 & $2 L_{\mathrm{S}}$ & $0.236 \mathrm{D}-06$ & & \\
\hline-12.936 & 170.64581 & 4.4555739318 & $5 L_{2}-5 L_{3}$ & $0.592 \mathrm{D}-08$ & 0.01018 & \\
\hline 11.279 & 142.54854 & 0.0251196097 & $2 v+\Omega_{2}+\Omega_{3}$ & $-0.908 \mathrm{D}-09$ & -0.00245 & \\
\hline-9.507 & 167.20958 & 0.0006493040 & $2 L_{\mathrm{S}}+2 \varpi_{4}+\Omega_{1}$ & $0.976 \mathrm{D}-07$ & & \\
\hline 9.446 & 11.38600 & 0.0246753155 & $2 v+2 \Omega_{2}$ & $-0.278 \mathrm{D}-10$ & -0.00026 & \\
\hline 8.857 & 57.05786 & 7.1289183312 & $4 L_{1}-4 L_{2}$ & $-0.310 \mathrm{D}-07$ & -0.00426 & \\
\hline 7.156 & 305.71256 & 0.0030233219 & $2 L_{\mathrm{S}}-\varpi_{3}-2 \Omega_{3}$ & $-0.402 \mathrm{D}-06$ & & \\
\hline-6.982 & 358.71911 & 2.7856729212 & $2 L_{2}-2 L_{4}$ & $0.342 \mathrm{D}-07$ & -0.03602 & \\
\hline 6.763 & 253.75671 & 0.0005529787 & $2 L_{\mathrm{S}}-\varpi_{4}+\Omega_{1}$ & $0.228 \mathrm{D}-06$ & & \\
\hline 6.539 & 156.48888 & 8.9111510231 & $5 L_{1}-5 L_{2}$ & $-0.315 \mathrm{D}-05$ & 4.82813 & \\
\hline-6.354 & 143.29880 & 0.0000934783 & $-\Omega_{3}+\Omega_{4}$ & $0.873 \mathrm{D}-06$ & 7.69205 & \\
\hline 6.116 & 90.32784 & 0.0252444417 & $2 v+\Omega_{2}+\Omega_{0}$ & $0.802 \mathrm{D}-07$ & -0.69407 & \\
\hline-5.888 & 88.10350 & 0.0156763933 & $2 L_{\mathrm{S}}+v-\varpi_{3}$ & $0.356 \mathrm{D}-05$ & & \\
\hline-5.265 & 333.05162 & 0.0259730692 & $2 v+\varpi_{3}+\varpi_{4}$ & $-0.172 \mathrm{D}-08$ & -0.01745 & \\
\hline-5.072 & 175.24631 & 0.0004325287 & $-\Psi+\varpi_{1}+\varpi_{3}-\Omega_{2}-\Omega_{3}$ & $0.211 \mathrm{D}-05$ & -28.70620 & * \\
\hline-4.469 & 112.24971 & 0.0229285674 & $2 v+\Omega_{1}+\Omega_{2}$ & $0.858 \mathrm{D}-06$ & -0.66994 & \\
\hline-4.421 & 106.67141 & 0.0260930584 & $2 v+2 \varpi_{3}$ & $-0.248 \mathrm{D}-04$ & 28.23524 & * \\
\hline-3.902 & 94.90412 & 6.2378035398 & $7 L_{2}-7 L_{3}$ & $-0.271 \mathrm{D}-07$ & 0.01426 & \\
\hline 3.740 & 226.69474 & 0.0025481216 & $\Psi-2 \varpi_{3}+2 \Omega_{3}$ & $-0.148 \mathrm{D}-05$ & 21.42465 & * \\
\hline-3.235 & 359.36045 & 1.3928364606 & $L_{2}-L_{4}$ & $0.171 \mathrm{D}-07$ & -0.01891 & \\
\hline 2.868 & 264.82632 & 10.6933779822 & $6 L_{1}-6 L_{2}$ & $-0.532 \mathrm{D}-06$ & 0.75409 & \\
\hline 2.831 & 77.40284 & 0.0030164436 & $L_{\mathrm{S}}+\Psi-2 \varpi_{2}-\varpi_{3}$ & $0.153 \mathrm{D}-05$ & & $*$ \\
\hline 2.531 & 292.40619 & 0.0252196582 & $2 v+\Omega_{2}+\Omega_{4}$ & $-0.570 \mathrm{D}-05$ & 1.13075 & \\
\hline
\end{tabular}


V. Lainey et al.: Synthetic representation of the Galilean satellites' orbital motions, Online Material p 4

Table 9. Solution for the variable $z_{2}$ (eccentricity and pericenter of Europa). The series is expressed in complex exponentials.

\begin{tabular}{|c|c|c|c|c|c|c|}
\hline $\begin{array}{r}\text { Amplitude } \\
(\mathrm{km})\end{array}$ & $\begin{array}{r}\text { Phase } \\
\text { (deg) }\end{array}$ & $\begin{array}{r}\text { Frequency } \\
\text { (rad/day) }\end{array}$ & Identification & $\begin{array}{r}\text { Freq. diff. } \\
\text { (rad/day) }\end{array}$ & $\begin{array}{r}\text { Ph. diff. } \\
\text { (deg) }\end{array}$ & \\
\hline-6282.273 & 234.33628 & -0.0129068641 & $-v$ & $0.000 \mathrm{D}+00$ & 0.00000 & \\
\hline 200.640 & 338.60239 & 1.7693227079 & $L_{2}$ & $0.318 \mathrm{D}-08$ & -0.00387 & \\
\hline 143.585 & 121.78957 & 0.0001272742 & $\varpi_{3}$ & $-0.538 \mathrm{D}-10$ & 0.00003 & \\
\hline 132.974 & 157.19194 & 0.0006779734 & $\varpi_{2}$ & $0.000 \mathrm{D}+00$ & 0.00000 & \\
\hline 81.249 & 319.93931 & 0.0000320566 & $\varpi_{4}$ & $0.848 \mathrm{D}-08$ & -0.02219 & \\
\hline 56.187 & 92.21491 & -0.9040216581 & $-2 L_{2}+3 L_{3}$ & $0.661 \mathrm{D}-08$ & -0.00999 & \\
\hline 55.280 & 82.86051 & 3.5515522950 & $L_{1}$ & $-0.880 \mathrm{D}-08$ & 0.00141 & \\
\hline-21.206 & 16.47324 & 0.8782079195 & $L_{3}$ & $0.407 \mathrm{D}-08$ & -0.00592 & \\
\hline-19.769 & 25.82779 & -3.5773660260 & $-3 L_{1}+4 L_{2}$ & $0.119 \mathrm{D}-07$ & -0.01948 & \\
\hline-18.725 & 130.08648 & -1.7951364497 & $-3 L_{2}+4 L_{3}$ & 0.107D-07 & -0.01275 & \\
\hline 9.731 & 167.95660 & -2.6862512422 & $-4 L_{2}+5 L_{3}$ & $0.157 \mathrm{D}-07$ & -0.01407 & \\
\hline 9.334 & 346.88341 & -0.0259410024 & $-2 v-\varpi_{3}$ & $-0.191 \mathrm{D}-10$ & -0.00046 & \\
\hline 7.275 & 339.88292 & -1.0163502160 & $-L_{2}+2 L_{4}$ & $-0.282 \mathrm{D}-07$ & 0.03251 & \\
\hline-5.516 & 281.57856 & -5.3595956184 & $-4 L_{1}+5 L_{2}$ & $0.293 \mathrm{D}-07$ & -0.03365 & \\
\hline 4.962 & 148.75621 & -0.0258457929 & $-2 v-\varpi_{4}$ & $-0.465 \mathrm{D}-09$ & -0.00078 & \\
\hline 4.203 & 356.68305 & -7.1418248394 & $-9 L_{2}+10 L_{3}$ & $-0.325 \mathrm{D}-06$ & 0.60349 & \\
\hline-3.488 & 311.42179 & -0.0264916967 & $-2 v-\varpi_{2}$ & $-0.500 \mathrm{D}-08$ & 0.05883 & \\
\hline 2.920 & 113.06959 & 0.3765422106 & $L_{4}-2 \Omega_{4}+2 \Omega_{0}$ & $0.514 \mathrm{D}-05$ & -1.61834 & \\
\hline 2.825 & 178.77781 & 4.4427230757 & $4 L_{2}-3 L_{3}-2 \Omega_{4}+2 \Omega_{0}$ & $0.512 \mathrm{D}-05$ & -1.59203 & \\
\hline 2.772 & 243.69827 & -4.4684808201 & $-6 L_{2}+7 L_{3}$ & 0.184D-07 & -0.01814 & \\
\hline-2.483 & 300.73159 & 2.6604375002 & $2 L_{2}-L_{3}$ & $-0.155 \mathrm{D}-08$ & -0.00188 & \\
\hline-1.836 & 72.96507 & -8.9240546532 & $-6 L_{1}+7 L_{2}$ & $-0.860 \mathrm{D}-07$ & 0.05304 & \\
\hline-1.803 & 43.31370 & 0.0158069532 & $2 L_{\mathrm{S}}+v-\varpi_{3}-\Omega_{3}$ & $-0.208 \mathrm{D}-05$ & & $*$ \\
\hline 1.549 & 111.37726 & 7.1160114825 & $7 L_{2}-6 L_{3}$ & $-0.462 \mathrm{D}-07$ & 0.00844 & \\
\hline 1.353 & 335.08976 & -2.4091843401 & $-2 L_{2}+3 L_{4}$ & $-0.238 \mathrm{D}-05$ & 5.48412 & \\
\hline-1.242 & 153.77170 & 0.0006823661 & $L_{\mathrm{S}}-\Psi-\varpi_{4}-\Omega_{1}$ & $-0.163 \mathrm{D}-06$ & & * \\
\hline 1.219 & 149.24800 & 6.2248966819 & $6 L_{2}-5 L_{3}$ & $-0.331 \mathrm{D}-07$ & 0.00651 & \\
\hline-1.201 & 328.79491 & -10.7062843289 & $-7 L_{1}+8 L_{2}$ & $0.146 \mathrm{D}-07$ & -0.04020 & \\
\hline 1.109 & 187.11938 & 5.3337818461 & $5 L_{2}-4 L_{3}$ & $0.152 \mathrm{D}-07$ & 0.00393 & \\
\hline-1.051 & 354.02690 & -0.0114535888 & $L_{\mathrm{S}}-v+\varpi_{3}+\Omega_{3}-\Omega_{0}$ & $-0.730 \mathrm{D}-06$ & & * \\
\hline
\end{tabular}

Table 10. Solution for the variable $\zeta_{2}$ (inclination and node of Europa). The series is expressed in complex exponentials.

\begin{tabular}{rrccrr}
\hline \hline $\begin{array}{r}\text { Amplitude } \\
(\mathrm{km})\end{array}$ & $\begin{array}{c}\text { Phase } \\
(\mathrm{deg})\end{array}$ & $\begin{array}{c}\text { Frequency } \\
(\mathrm{rad} / \mathrm{day})\end{array}$ & Identification & $\begin{array}{r}\text { Freq. diff. } \\
(\mathrm{rad} / \mathrm{day})\end{array}$ & $\begin{array}{r}\text { Ph. diff. } \\
(\mathrm{deg})\end{array}$ \\
\hline 2712.225 & 60.02915 & -0.0005692064 & $\Omega_{2}$ & $0.000 \mathrm{D}+00$ & 0.00000 \\
147.706 & 191.18947 & -0.0001249131 & $\Omega_{3}$ & $0.249 \mathrm{D}-11$ & 0.00003 \\
111.600 & 138.28257 & 0.0000000000 & $\Omega_{0}$ & $0.000 \mathrm{D}+00$ & -0.00539 \\
39.623 & 342.17000 & -0.0000305616 & $\Omega_{4}$ & $0.347 \mathrm{D}-09$ & 0.01035 \\
-7.050 & 160.22752 & -0.0023150966 & $\Omega_{1}$ & $0.514 \mathrm{D}-09$ & -0.00434 \\
-6.910 & 48.64343 & -0.0252445219 & $-2 v-\Omega_{2}$ & $0.137 \mathrm{D}-10$ & -0.00002 \\
4.873 & 318.58591 & 0.0029003677 & $2 L_{\mathrm{S}}$ & $-0.173 \mathrm{D}-09$ & \\
1.235 & 16.31813 & -0.0014500579 & $-L_{\mathrm{S}}$ & $-0.126 \mathrm{D}-06$ & \\
\hline
\end{tabular}


V. Lainey et al.: Synthetic representation of the Galilean satellites' orbital motions, Online Material p 5

Table 11. Solution for the variable $a_{3}$ (semi-major axis of Ganymede). The series is expressed in cosine.

\begin{tabular}{rrccrr}
\hline \hline $\begin{array}{r}\text { Amplitude } \\
(\mathrm{km})\end{array}$ & $\begin{array}{r}\text { Phase } \\
(\mathrm{deg})\end{array}$ & $\begin{array}{c}\text { Frequency } \\
(\mathrm{rad} / \text { day })\end{array}$ & Identification & $\begin{array}{r}\text { Freq. diff. } \\
(\mathrm{rad} / \text { day })\end{array}$ & $\begin{array}{r}\text { Ph. diff. } \\
(\mathrm{deg})\end{array}$ \\
\hline 1070621.016 & 0.00000 & 0.0000000000 & & & \\
208.394 & 66.38716 & 2.6733443704 & $L_{1}-L_{3}$ & $-0.783 \mathrm{D}-08$ & 0.00744 \\
96.488 & 322.12917 & 0.8911147889 & $L_{2}-L_{3}$ & $-0.134 \mathrm{D}-08$ & 0.00203 \\
34.378 & 74.46116 & 1.0034433457 & $2 L_{3}-2 L_{4}$ & $0.346 \mathrm{D}-07$ & -0.04047 \\
-18.272 & 284.25832 & 1.7822295778 & $2 L_{2}-2 L_{3}$ & $-0.269 \mathrm{D}-08$ & 0.00408 \\
16.393 & 111.65062 & 1.5051650462 & $3 L_{3}-3 L_{4}$ & $0.243 \mathrm{D}-07$ & -0.01958 \\
10.493 & 37.22994 & 0.5017216696 & $L_{3}-L_{4}$ & $0.205 \mathrm{D}-07$ & -0.01959 \\
8.196 & 148.92348 & 2.0068866946 & $4 L_{3}-4 L_{4}$ & $0.661 \mathrm{D}-07$ & -0.08209 \\
-5.904 & 132.77458 & 5.3466887181 & $6 L_{2}-6 L_{3}$ & $0.710 \mathrm{D}-08$ & 0.01261 \\
-5.434 & 208.51658 & 3.5644591656 & $4 L_{2}-4 L_{3}$ & $-0.155 \mathrm{D}-07$ & 0.00821 \\
4.353 & 115.29854 & 1.7535157350 & $2 L_{3}-2 L_{\mathrm{S}}$ & $-0.255 \mathrm{D}-06$ & 0.43003 \\
4.207 & 186.15405 & 2.5086083722 & $5 L_{3}-5 L_{4}$ & $0.786 \mathrm{D}-07$ & -0.10232 \\
-3.111 & 170.64579 & 4.4555739318 & $5 L_{2}-5 L_{3}$ & $0.592 \mathrm{D}-08$ & 0.01020 \\
2.198 & 223.38618 & 3.0103300418 & $6 L_{3}-6 L_{4}$ & $0.991 \mathrm{D}-07$ & -0.12410 \\
-1.794 & 94.90455 & 6.2378035398 & $7 L_{2}-7 L_{3}$ & $-0.271 \mathrm{D}-07$ & 0.01383 \\
1.677 & 247.45283 & 0.0130341383 & $v+\varpi_{3}$ & $-0.839 \mathrm{D}-11$ & 0.00049 \\
-1.639 & 282.88546 & 0.0135848349 & $v+\varpi_{2}$ & $0.264 \mathrm{D}-08$ & -0.02980 \\
1.494 & 55.40543 & 0.6269963378 & $2 L_{3}-3 L_{4}+\varpi_{4}$ & $-0.713 \mathrm{D}-05$ & -0.32458 \\
1.162 & 260.61772 & 3.5120517183 & $7 L_{3}-7 L_{4}$ & $0.113 \mathrm{D}-06$ & -0.14529 \\
1.109 & 9.24780 & 0.1253136166 & $L_{3}-2 L_{4}+\varpi_{4}$ & $-0.461 \mathrm{D}-04$ & 8.62270 \\
$\ldots$ & & & & & \\
0.364 & 112.32745 & 0.1123285451 & $-L_{2}+3 L_{3}-2 L_{4}$ & $0.476 \mathrm{D}-07$ & -0.03795 \\
\hline
\end{tabular}

Table 12. Solution for $\mathcal{L}_{3}$ (mean longitude of Ganymede): $\mathcal{L}_{3}=L_{3}+$ series expressed in sine, with $L_{3}=0.2874089391143348+0.878207923589 \times$ $T$.

\begin{tabular}{|c|c|c|c|c|c|c|}
\hline $\begin{array}{r}\text { Amplitude } \\
(\mathrm{km})\end{array}$ & $\begin{array}{r}\text { Phase } \\
\text { (deg) }\end{array}$ & $\begin{array}{r}\text { Frequency } \\
\text { (rad/day) }\end{array}$ & Identification & $\begin{array}{r}\text { Freq. diff. } \\
\text { (rad/day) }\end{array}$ & $\begin{array}{r}\text { Ph. diff. } \\
\text { (deg) }\end{array}$ & \\
\hline 247.399 & 122.63008 & 0.0014500978 & $L_{\mathrm{S}}$ & $0.859 \mathrm{D}-07$ & & \\
\hline-195.778 & 247.45287 & 0.0130341383 & $v+\varpi_{3}$ & $-0.568 \mathrm{D}-11$ & 0.00045 & \\
\hline 161.918 & 282.88704 & 0.0135848348 & $v+\varpi_{2}$ & $0.276 \mathrm{D}-08$ & -0.03138 & \\
\hline-124.590 & 246.38709 & 2.6733443704 & $3 L_{2}-3 L_{3}$ & $-0.782 \mathrm{D}-08$ & 0.00650 & \\
\hline-102.295 & 85.58049 & 0.0129389288 & $v+\varpi_{4}$ & $0.447 \mathrm{D}-09$ & 0.00036 & \\
\hline 87.282 & 322.12912 & 0.8911147889 & $L_{2}-L_{3}$ & $-0.134 \mathrm{D}-08$ & 0.00208 & \\
\hline-85.780 & 74.46115 & 1.0034433457 & $2 L_{3}-2 L_{4}$ & $0.346 \mathrm{D}-07$ & -0.04046 & \\
\hline-64.989 & 37.23009 & 0.5017216704 & $L_{3}-L_{4}$ & $0.197 \mathrm{D}-07$ & -0.01974 & \\
\hline 58.233 & 160.01306 & 0.0000298809 & $-\varpi_{3}+\varpi_{4}-\Omega_{3}+\Omega_{0}$ & $-0.177 \mathrm{D}-06$ & -14.79786 & \\
\hline-52.381 & 307.74553 & 0.0001249528 & $-\Omega_{3}+\Omega_{0}$ & $-0.397 \mathrm{D}-07$ & -0.65785 & \\
\hline-45.777 & 104.25834 & 1.7822295778 & $L_{1}-L_{2}$ & $-0.270 \mathrm{D}-08$ & 0.00506 & \\
\hline-32.907 & 111.71745 & 1.5051650065 & $3 L_{3}-3 L_{4}$ & $0.640 \mathrm{D}-07$ & -0.08641 & \\
\hline-18.176 & 109.31254 & 0.0030507678 & $\Psi$ & $0.244 \mathrm{D}-06$ & 0.00706 & \\
\hline 16.565 & 326.08583 & 0.0006516407 & $-\rho-4 \varpi_{4}$ & $-0.391 \mathrm{D}-07$ & -0.35748 & \\
\hline-15.553 & 10.80820 & 0.1253082718 & $L_{3}-2 L_{4}+\varpi_{4}$ & $-0.407 \mathrm{D}-04$ & 7.06230 & * \\
\hline-14.523 & 160.02848 & 0.0005566368 & $-\rho-\varpi_{3}-3 \varpi_{4}$ & $-0.244 \mathrm{D}-06$ & 3.82739 & \\
\hline-14.416 & 148.92304 & 2.0068866946 & $4 L_{3}-4 L_{4}$ & $0.661 \mathrm{D}-07$ & -0.08165 & \\
\hline 10.227 & 132.77449 & 5.3466887181 & $6 L_{2}-6 L_{3}$ & $0.710 \mathrm{D}-08$ & 0.01270 & \\
\hline 9.417 & 208.51667 & 3.5644591656 & $4 L_{2}-4 L_{3}$ & $-0.155 \mathrm{D}-07$ & 0.00812 & \\
\hline 8.079 & 306.87973 & 0.0000924270 & $-2 L_{\mathrm{S}}+\varpi_{2}-\Omega_{1}-\Omega_{0}$ & $0.275 \mathrm{D}-06$ & & * \\
\hline-7.617 & 115.28233 & 1.7535157644 & $2 L_{3}-2 L_{\mathrm{S}}$ & $-0.285 \mathrm{D}-06$ & 0.44624 & \\
\hline 6.868 & 88.95953 & 0.0029001310 & $2 L_{\mathrm{S}}$ & $0.237 \mathrm{D}-06$ & & \\
\hline-6.769 & 186.15469 & 2.5086083722 & $5 L_{3}-5 L_{4}$ & $0.786 \mathrm{D}-07$ & -0.10296 & \\
\hline-6.056 & 142.44956 & 0.0004483462 & $-L_{\mathrm{S}}-\Psi+\varpi_{1}-\Omega_{1}+\Omega_{4}$ & $-0.754 \mathrm{D}-06$ & & * \\
\hline 5.628 & 11.38555 & 0.0246753155 & $2 v+2 \Omega_{2}$ & $-0.191 \mathrm{D}-10$ & 0.00019 & \\
\hline 5.034 & 170.64584 & 4.4555739318 & $5 L_{2}-5 L_{3}$ & $0.592 \mathrm{D}-08$ & 0.01015 & \\
\hline-5.032 & 55.35749 & 0.6269971274 & $2 L_{3}-3 L_{4}+\varpi_{4}$ & $-0.792 \mathrm{D}-05$ & -0.27664 & \\
\hline-4.985 & 206.98165 & 0.0004363323 & $L_{\mathrm{S}}+2 \Omega_{2}-\Omega_{3}+\Omega_{0}$ & $0.352 \mathrm{D}-06$ & & * \\
\hline-4.534 & 112.32691 & 0.1123285428 & $-L_{2}+3 L_{3}-2 L_{4}$ & $0.500 \mathrm{D}-07$ & -0.03741 & \\
\hline-4.149 & 129.60090 & 0.0251466639 & $-L_{\mathrm{S}}+2 v-\rho$ & $-0.326 \mathrm{D}-05$ & & $*$ \\
\hline-3.488 & 325.79427 & 0.0017074577 & $L_{\mathrm{S}}-\rho-\varpi_{2}-\Omega_{3}-\Omega_{4}$ & $0.890 \mathrm{D}-07$ & & * \\
\hline
\end{tabular}


V. Lainey et al.: Synthetic representation of the Galilean satellites' orbital motions, Online Material p 6

Table 13. Solution for the variable $z_{3}$ (eccentricity and pericenter of Ganymede). The series is expressed in complex exponentials.

\begin{tabular}{|c|c|c|c|c|c|c|}
\hline $\begin{array}{r}\text { Amplitude } \\
(\mathrm{km})\end{array}$ & $\begin{array}{r}\text { Phase } \\
(\text { deg) }\end{array}$ & $\begin{array}{r}\text { Frequency } \\
\text { (rad/day) }\end{array}$ & Identification & $\begin{array}{r}\text { Freq. diff. } \\
\text { (rad/day) }\end{array}$ & $\begin{array}{r}\text { Ph. diff. } \\
\text { (deg) }\end{array}$ & \\
\hline 1529.897 & 121.78960 & 0.0001272741 & $\varpi_{3}$ & $0.000 \mathrm{D}+00$ & 0.00000 & \\
\hline 825.549 & 319.91861 & 0.0000320643 & $\varpi_{4}$ & $0.758 \mathrm{D}-09$ & -0.00149 & \\
\hline 634.441 & 234.33628 & -0.0129068641 & $-v$ & $0.000 \mathrm{D}+00$ & 0.00000 & \\
\hline 219.006 & 302.02716 & -0.1252354408 & $-L_{3}+2 L_{4}$ & $-0.160 \mathrm{D}-07$ & 0.01947 & \\
\hline 191.119 & 16.46862 & 0.8782079244 & $L_{3}$ & $-0.836 \mathrm{D}-09$ & -0.00130 & \\
\hline 121.194 & 82.86189 & 3.5515522950 & $L_{1}$ & $-0.880 \mathrm{D}-08$ & 0.00003 & \\
\hline-70.530 & 130.07531 & -1.7951364395 & $-3 L_{2}+4 L_{3}$ & $0.441 \mathrm{D}-09$ & -0.00158 & \\
\hline 53.216 & 338.59968 & 1.7693227129 & $L_{2}$ & $-0.181 \mathrm{D}-08$ & -0.00116 & \\
\hline-33.873 & 92.20641 & -0.9040216503 & $-2 L_{2}+3 L_{3}$ & $-0.119 \mathrm{D}-08$ & -0.00149 & \\
\hline 30.813 & 264.80574 & -0.6269571234 & $-2 L_{3}+3 L_{4}$ & $-0.235 \mathrm{D}-07$ & 0.03054 & \\
\hline-19.458 & 339.25199 & 0.3764862399 & $L_{4}$ & $-0.648 \mathrm{D}-08$ & 0.00498 & \\
\hline 11.301 & 227.57993 & -1.1286788041 & $-3 L_{3}+4 L_{4}$ & $-0.329 \mathrm{D}-07$ & 0.04600 & \\
\hline-7.581 & 346.88325 & -0.0259410024 & $-2 v-\varpi_{3}$ & $-0.840 \mathrm{D}-11$ & -0.00030 & \\
\hline-7.580 & 160.30462 & 0.0006777426 & $\varpi_{2}$ & $0.231 \mathrm{D}-06$ & -3.11268 & \\
\hline-6.536 & 81.08319 & -0.8753076942 & $-L_{3}+\varpi_{1}+\varpi_{3}-\Omega_{3}+\Omega_{0}$ & $0.162 \mathrm{D}-04$ & -3.38447 & $*$ \\
\hline-6.168 & 243.68203 & -4.4684807883 & $-6 L_{2}+7 L_{3}$ & $-0.133 \mathrm{D}-07$ & -0.00190 & \\
\hline-6.136 & 167.94426 & -2.6862512193 & $-4 L_{2}+5 L_{3}$ & $-0.728 \mathrm{D}-08$ & -0.00173 & \\
\hline 5.171 & 155.49097 & 0.0027731330 & $2 L_{\mathrm{S}}-\varpi_{3}$ & $-0.396 \mathrm{D}-07$ & -0.07450 & \\
\hline 4.990 & 190.35365 & -1.6304004832 & $-4 L_{3}+5 L_{4}$ & $-0.440 \mathrm{D}-07$ & 0.06193 & \\
\hline-4.084 & 148.75671 & -0.0258457930 & $-2 v-\varpi_{4}$ & $-0.437 \mathrm{D}-09$ & -0.00128 & \\
\hline 3.745 & 90.90874 & 1.8816512921 & $3 L_{3}-2 L_{4}$ & $0.118 \mathrm{D}-07$ & -0.02073 & \\
\hline-3.222 & 205.81317 & -3.5773660056 & $-5 L_{2}+6 L_{3}$ & $-0.845 \mathrm{D}-08$ & -0.00184 & \\
\hline-2.648 & 306.31315 & 0.2512257684 & $-L_{3}+3 L_{4}-\varpi_{3}-\Omega_{3}+\Omega_{4}$ & $-0.791 \mathrm{D}-05$ & 4.19169 & \\
\hline 2.614 & 269.57365 & -0.2504961383 & $-2 L_{3}+4 L_{4}-\varpi_{3}-\Omega_{3}+\Omega_{4}$ & $-0.770 \mathrm{D}-05$ & 3.72084 & \\
\hline 2.588 & 197.71646 & 0.0000000000 & $\varpi_{3}+\Omega_{3}-\Omega_{0}$ & $-0.236 \mathrm{D}-05$ & 23.01454 & $*$ \\
\hline 2.478 & 317.33703 & 0.0028683339 & $L_{\mathrm{S}}+2 \varpi_{2}-2 \Omega_{4}$ & $-0.108 \mathrm{D}-05$ & & $*$ \\
\hline 2.425 & 318.61041 & 0.0014501893 & $L_{\mathrm{S}}$ & $-0.555 \mathrm{D}-08$ & -0.00737 & \\
\hline 2.382 & 153.12540 & -2.1321221655 & $-5 L_{3}+6 L_{4}$ & $-0.519 \mathrm{D}-07$ & 0.07984 & \\
\hline 2.243 & 128.05821 & 2.3833730193 & $4 L_{3}-3 L_{4}$ & $-0.252 \mathrm{D}-07$ & 0.04015 & \\
\hline-1.503 & 53.69648 & 1.3799296042 & $2 L_{3}-L_{4}$ & $0.956 \mathrm{D}-08$ & -0.01881 & \\
\hline 1.278 & 165.36674 & 2.8850946416 & $5 L_{3}-4 L_{4}$ & $0.426 \mathrm{D}-07$ & -0.05803 & \\
\hline-1.197 & 281.55100 & -5.3595955727 & $-4 L_{1}+5 L_{2}$ & $-0.164 \mathrm{D}-07$ & -0.00609 & \\
\hline 1.186 & 115.89526 & -2.6338438454 & $-6 L_{3}+7 L_{4}$ & $-0.621 \mathrm{D}-07$ & 0.09963 & \\
\hline-1.110 & 23.33241 & -0.8738575909 & $-L_{3}+\varpi_{1}+\varpi_{2}-2 \Omega_{2}+\Omega_{3}+\Omega_{0}$ & $0.539 \mathrm{D}-05$ & -7.91065 & $*$ \\
\hline
\end{tabular}

Table 14. Solution for the variable $\zeta_{3}$ (inclination and node of Ganymede). The series is expressed in complex exponentials.

\begin{tabular}{rcccrr}
\hline \hline $\begin{array}{r}\text { Amplitude } \\
(\mathrm{km})\end{array}$ & $\begin{array}{c}\text { Phase } \\
(\mathrm{deg})\end{array}$ & $\begin{array}{c}\text { Frequency } \\
(\mathrm{rad} / \mathrm{day})\end{array}$ & Identification & $\begin{array}{r}\text { Freq. diff. } \\
(\mathrm{rad} / \mathrm{day})\end{array}$ & $\begin{array}{r}\text { Ph. diff. } \\
(\mathrm{deg})\end{array}$ \\
\hline 1705.791 & 191.18950 & -0.0001249131 & $\Omega_{3}$ & $0.000 \mathrm{D}+00$ & 0.00000 \\
913.571 & 138.27696 & 0.0000000000 & $\Omega_{0}$ & $0.000 \mathrm{D}+00$ & 0.00022 \\
376.146 & 342.17492 & -0.0000305610 & $\Omega_{4}$ & $-0.238 \mathrm{D}-09$ & 0.00543 \\
-154.376 & 60.02914 & -0.0005692063 & $\Omega_{2}$ & $-0.842 \mathrm{D}-10$ & 0.00001 \\
16.841 & 318.58769 & 0.0029003665 & $2 L_{\mathrm{S}}$ & $0.997 \mathrm{D}-09$ & \\
2.694 & 16.31649 & -0.0014500554 & $-L_{\mathrm{S}}$ & $-0.128 \mathrm{D}-06$ & \\
2.188 & 101.14211 & 0.0014501384 & $L_{\mathrm{S}}$ & $0.454 \mathrm{D}-07$ & \\
1.921 & 261.09101 & 0.0043504622 & $3 L_{\mathrm{S}}$ & $0.891 \mathrm{D}-07$ & \\
1.458 & 48.64347 & -0.0252445219 & $-2 v-\Omega_{2}$ & $0.127 \mathrm{D}-10$ & -0.00006 \\
\hline
\end{tabular}


V. Lainey et al.: Synthetic representation of the Galilean satellites' orbital motions, Online Material p 7

Table 15. Solution for the variable $a_{4}$ (semi-major axis of Callisto). The series is expressed in cosine.

\begin{tabular}{rrccrr}
\hline \hline $\begin{array}{r}\text { Amplitude } \\
(\mathrm{km})\end{array}$ & $\begin{array}{r}\text { Phase } \\
(\mathrm{deg})\end{array}$ & $\begin{array}{c}\text { Frequency } \\
(\mathrm{rad} / \mathrm{day})\end{array}$ & Identification & $\begin{array}{r}\text { Freq. diff. } \\
\text { (rad/day) }\end{array}$ & $\begin{array}{r}\text { Ph. diff. } \\
(\mathrm{deg})\end{array}$ \\
\hline 1883133.534 & 0.00000 & 0.0000000000 & & & \\
537.835 & 37.22265 & 0.5017216817 & $L_{3}-L_{4}$ & $0.851 \mathrm{D}-08$ & -0.01230 \\
412.594 & 103.61504 & 3.1750660413 & $L_{1}-L_{4}$ & $0.114 \mathrm{D}-07$ & -0.01009 \\
192.606 & 359.35287 & 1.3928364698 & $L_{2}-L_{4}$ & $0.785 \mathrm{D}-08$ & -0.01133 \\
-62.438 & 74.43094 & 1.0034433697 & $2 L_{3}-2 L_{4}$ & $0.106 \mathrm{D}-07$ & -0.01025 \\
41.749 & 40.92573 & 0.7500722587 & $2 L_{4}-2 L_{\mathrm{S}}$ & $-0.159 \mathrm{D}-06$ & 0.38214 \\
-29.893 & 111.66879 & 1.5051650462 & $3 L_{3}-3 L_{4}$ & $0.243 \mathrm{D}-07$ & -0.03775 \\
-14.977 & 148.89551 & 2.0068867266 & $4 L_{3}-4 L_{4}$ & $0.340 \mathrm{D}-07$ & -0.05412 \\
-7.689 & 186.12419 & 2.5086084022 & $5 L_{3}-5 L_{4}$ & $0.485 \mathrm{D}-07$ & -0.07246 \\
-7.116 & 278.66101 & 0.7486221659 & $2 L_{4}-3 L_{\mathrm{S}}$ & $-0.250 \mathrm{D}-06$ & \\
5.210 & 8.64176 & 0.3764591707 & $L_{4}-\varpi_{4}$ & $-0.500 \mathrm{D}-05$ & 10.69809 \\
4.246 & 296.06433 & 0.1253067807 & $L_{3}-2 L_{4}+\varpi_{4}-\Omega_{4}+\Omega_{0}$ & $0.870 \mathrm{D}-05$ & 32.09690 \\
-3.938 & 191.93984 & 3.0103491233 & $6 L_{3}-6 L_{4}$ & $-0.190 \mathrm{D}-04$ & 31.32224 \\
3.577 & 249.65788 & 0.6269823880 & $-L_{3}+4 L_{4}-L_{\mathrm{S}}$ & $-0.696 \mathrm{D}-03$ & \\
3.291 & 86.37571 & 2.7986109087 & $L_{1}-2 L_{4}+\varpi_{4}$ & $0.976 \mathrm{D}-06$ & -2.11061 \\
-2.560 & 358.71376 & 2.7856729335 & $2 L_{2}-2 L_{4}$ & $0.219 \mathrm{D}-07$ & -0.03067 \\
-2.124 & 260.58738 & 3.5120517575 & $7 L_{3}-7 L_{4}$ & $0.736 \mathrm{D}-07$ & -0.11495 \\
-1.795 & 106.47783 & 1.1287042579 & $3 L_{4}-2 \varpi_{2}+\varpi_{4}-\Omega_{2}-\Omega_{0}$ & $-0.233 \mathrm{D}-06$ & -1.47999 \\
1.622 & 314.39993 & 6.7395238593 & $3 L_{1}-2 L_{2}-L_{4}$ & $0.134 \mathrm{D}-05$ & -2.26818 \\
1.619 & 342.47195 & 1.0163811590 & $L_{2}-2 L_{4}+\varpi_{3}-2 \varpi_{4}+\Omega_{4}+\Omega_{0}$ & $0.167 \mathrm{D}-05$ & 0.02550 \\
$\ldots$ & & & & & \\
0.037 & 325.97902 & 0.0006516502 & $-\rho-4 \varpi_{4}$ & $-0.486 \mathrm{D}-07$ & -0.25067 \\
-0.028 & 163.85638 & 0.0005563954 & $-\rho-\varpi_{3}-3 \varpi_{4}$ & $-0.285 \mathrm{D}-08$ & -0.00051 \\
\hline & & & & & \\
\hline
\end{tabular}

Table 16. Solution for $\mathcal{L}_{4}$ (mean longitude of Callisto): $\mathcal{L}_{4}=L_{4}+$ series expressed in sine, with $L_{4}=0.3620341291375704+0.376486233434 \times T$.

\begin{tabular}{rrccrr}
\hline \hline $\begin{array}{r}\text { Amplitude } \\
(\mathrm{km})\end{array}$ & $\begin{array}{r}\text { Phase } \\
(\mathrm{deg})\end{array}$ & $\begin{array}{c}\text { Frequency } \\
(\mathrm{rad} / \mathrm{day})\end{array}$ & Identification & $\begin{array}{r}\text { Freq. diff. } \\
\text { (rad/day) }\end{array}$ & $\begin{array}{r}\text { Ph. diff. } \\
(\mathrm{deg})\end{array}$ \\
\hline 1051.926 & 122.63707 & 0.0014500979 & $L_{\mathrm{S}}$ & $0.858 \mathrm{D}-07$ & \\
-716.686 & 156.75463 & 0.0000297297 & $-\varpi_{3}+\varpi_{4}-\Omega_{3}+\Omega_{0}$ & $-0.256 \mathrm{D}-07$ & -11.53943 \\
415.260 & 37.23060 & 0.5017216724 & $L_{3}-L_{4}$ & $0.177 \mathrm{D}-07$ & -0.02025 \\
353.633 & 103.61820 & 3.1750660413 & $L_{1}-L_{4}$ & $0.114 \mathrm{D}-07$ & -0.01325 \\
144.421 & 359.35978 & 1.3928364637 & $L_{2}-L_{4}$ & $0.140 \mathrm{D}-07$ & -0.01824 \\
140.681 & 74.46111 & 1.0034433457 & $2 L_{3}-2 L_{4}$ & $0.346 \mathrm{D}-07$ & -0.04042 \\
-73.126 & 40.84572 & 0.7500723697 & $2 L_{4}-2 L_{\mathrm{S}}$ & $-0.270 \mathrm{D}-06$ & 0.46215 \\
63.092 & 307.75076 & 0.0001249401 & $-\Omega_{3}+\Omega_{0}$ & $-0.270 \mathrm{D}-07$ & -0.66308 \\
55.182 & 111.69205 & 1.5051650209 & $3 L_{3}-3 L_{4}$ & $0.496 \mathrm{D}-07$ & -0.06101 \\
35.015 & 83.82945 & 0.0029001339 & $2 L_{\mathrm{S}}$ & $0.234 \mathrm{D}-06$ & \\
-32.096 & 325.97507 & 0.0006516504 & $-\rho-4 \varpi_{4}$ & $-0.488 \mathrm{D}-07$ & -0.24672 \\
28.509 & 164.72259 & 0.0005564607 & $-\rho-\varpi_{3}-3 \varpi_{4}$ & $-0.681 \mathrm{D}-07$ & -0.86672 \\
-28.026 & 190.91569 & 0.1253079008 & $L_{3}-2 L_{4}+\varpi_{4}-\Omega_{4}+\Omega_{0}$ & $-0.982 \mathrm{D}-05$ & -16.94836 \\
24.467 & 148.92304 & 2.0068866946 & $4 L_{3}-4 L_{4}$ & $0.661 \mathrm{D}-07$ & -0.08165 \\
-21.866 & 321.95798 & 0.0000931663 & $-L_{\mathrm{S}}-\varpi_{2}-\Omega_{1}+\Omega_{3}-\Omega_{4}$ & $-0.579 \mathrm{D}-06$ & \\
12.469 & 278.25671 & 0.7486228617 & $2 L_{4}-3 L_{\mathrm{S}}$ & $-0.946 \mathrm{D}-06$ & \\
12.313 & 203.85907 & 0.0016550514 & $3 L_{\mathrm{S}}-\varpi_{1}-\varpi_{4}$ & $-0.819 \mathrm{D}-06$ & \\
11.596 & 186.15420 & 2.5086083722 & $5 L_{3}-5 L_{4}$ & $0.786 \mathrm{D}-07$ & -0.10247 \\
8.813 & 55.35470 & 0.6269971662 & $2 L_{3}-3 L_{4}+\varpi_{4}$ & $-0.795 \mathrm{D}-05$ & -0.27385 \\
\hline
\end{tabular}


V. Lainey et al.: Synthetic representation of the Galilean satellites' orbital motions, Online Material p 8

Table 17. Solution for the variable $z_{4}$ (eccentricity and pericenter of Callisto). The series is expressed in complex exponentials.

\begin{tabular}{|c|c|c|c|c|c|c|}
\hline $\begin{array}{r}\text { Amplitude } \\
(\mathrm{km})\end{array}$ & $\begin{array}{l}\text { Phase } \\
\text { (deg) }\end{array}$ & $\begin{array}{r}\text { Frequency } \\
\text { (rad/day) }\end{array}$ & Identification & $\begin{array}{r}\text { Freq. diff. } \\
\text { (rad/day) }\end{array}$ & $\begin{array}{r}\text { Ph. diff. } \\
\text { (deg) }\end{array}$ & \\
\hline 13889.204 & 319.91712 & 0.0000320651 & $\varpi_{4}$ & $0.000 \mathrm{D}+00$ & 0.00000 & \\
\hline 389.041 & 339.24735 & 0.3764862419 & $L_{4}$ & $-0.851 \mathrm{D}-08$ & 0.00962 & \\
\hline 299.394 & 16.46910 & 0.8782079244 & $L_{3}$ & $-0.836 \mathrm{D}-09$ & -0.00178 & \\
\hline-293.982 & 121.79592 & 0.0001272744 & $\varpi_{3}$ & $-0.283 \mathrm{D}-09$ & -0.00632 & \\
\hline 279.842 & 82.86193 & 3.5515522950 & $L_{1}$ & $-0.880 \mathrm{D}-08$ & -0.00001 & \\
\hline 119.593 & 338.59974 & 1.7693227129 & $L_{2}$ & $-0.181 \mathrm{D}-08$ & -0.00122 & \\
\hline 112.866 & 235.63186 & -2.7985797955 & $-L_{1}+2 L_{4}$ & $-0.239 \mathrm{D}-07$ & 0.02017 & \\
\hline 101.814 & 317.36333 & 0.0028683408 & $2 L_{\mathrm{S}}-\varpi_{4}$ & $-0.384 \mathrm{D}-07$ & -0.07438 & \\
\hline-92.198 & 264.81049 & -0.6269571253 & $-2 L_{3}+3 L_{4}$ & $-0.216 \mathrm{D}-07$ & 0.02579 & \\
\hline 62.837 & 298.32179 & -0.3735860173 & $-L_{4}+2 L_{\mathrm{S}}$ & $0.151 \mathrm{D}-06$ & -0.37269 & \\
\hline 55.709 & 339.89402 & -1.0163502275 & $-L_{2}+2 L_{4}$ & $-0.167 \mathrm{D}-07$ & 0.02141 & \\
\hline 55.049 & 301.99244 & -0.1252354245 & $-L_{3}+2 L_{4}$ & $-0.322 \mathrm{D}-07$ & 0.05419 & \\
\hline 37.208 & 190.89675 & 0.0000000000 & $\varpi_{4}+\Omega_{4}-\Omega_{0}$ & $-0.150 \mathrm{D}-05$ & 27.07647 & * \\
\hline-34.565 & 227.58138 & -1.1286788041 & $-3 L_{3}+4 L_{4}$ & $-0.329 \mathrm{D}-07$ & 0.04455 & \\
\hline 17.026 & 318.60304 & 0.0014501837 & $L_{\mathrm{S}}$ & $0.000 \mathrm{D}+00$ & 0.00000 & \\
\hline-15.439 & 190.35557 & -1.6304004832 & $-4 L_{3}+5 L_{4}$ & $-0.440 \mathrm{D}-07$ & 0.06001 & \\
\hline-11.375 & 80.04373 & 0.0043191832 & $3 L_{\mathrm{S}}+\Omega_{4}-\Omega_{0}$ & $0.807 \mathrm{D}-06$ & -0.33145 & \\
\hline 10.714 & 240.59046 & -0.3721359266 & $-L_{4}+3 L_{\mathrm{S}}$ & $0.244 \mathrm{D}-06$ & & \\
\hline-7.614 & 269.33833 & -0.2504960289 & $-2 L_{3}+4 L_{4}-\varpi_{4}$ & $-0.695 \mathrm{D}-05$ & 14.83780 & \\
\hline-7.420 & 153.12774 & -2.1321221655 & $-5 L_{3}+6 L_{4}$ & $-0.519 \mathrm{D}-07$ & 0.07750 & \\
\hline 6.949 & 20.17257 & 1.1265585019 & $3 L_{4}-2 L_{\mathrm{S}}$ & $-0.169 \mathrm{D}-06$ & 0.39227 & \\
\hline-5.377 & 318.57178 & -0.0000315849 & $\varpi_{4}+\Omega_{3}-2 \Omega_{4}+\Omega_{0}$ & $-0.141 \mathrm{D}-06$ & 6.45133 & \\
\hline-5.007 & 14.87965 & 0.0014182026 & $L_{\mathrm{S}}-\varpi_{4}$ & $-0.839 \mathrm{D}-07$ & & \\
\hline-3.712 & 115.89802 & -2.6338438454 & $-6 L_{3}+7 L_{4}$ & $-0.621 \mathrm{D}-07$ & 0.09687 & \\
\hline 3.639 & 294.60685 & 0.2512311791 & $-L_{3}+3 L_{4}-\varpi_{4}$ & $-0.125 \mathrm{D}-04$ & 26.77962 & * \\
\hline-3.516 & 53.67176 & 1.3799296163 & $2 L_{3}-L_{4}$ & $-0.256 \mathrm{D}-08$ & 0.00591 & \\
\hline 3.171 & 348.33561 & 0.7529452084 & $2 L_{4}-\varpi_{3}-\Omega_{3}+\Omega_{4}$ & $-0.566 \mathrm{D}-05$ & -0.62042 & \\
\hline-3.144 & 90.91586 & 1.8816512864 & $3 L_{3}-2 L_{4}$ & $0.175 \mathrm{D}-07$ & -0.02785 & \\
\hline 3.073 & 262.35471 & 0.0014822429 & $L_{\mathrm{S}}+\varpi_{4}$ & $0.593 \mathrm{D}-08$ & & \\
\hline-3.043 & 17.27883 & -0.0014180284 & $-L_{\mathrm{S}}+\varpi_{4}$ & $-0.902 \mathrm{D}-07$ & & \\
\hline-2.643 & 340.52894 & -2.4091866865 & $-2 L_{2}+3 L_{4}$ & $-0.354 \mathrm{D}-07$ & 0.04494 & \\
\hline-2.265 & 155.48788 & 0.0027731373 & $2 L_{\mathrm{S}}-\varpi_{3}$ & $-0.439 \mathrm{D}-07$ & -0.07141 & \\
\hline-2.214 & 232.51257 & -0.7522178950 & $-2 L_{4}+2 \varpi_{2}-\varpi_{4}+\Omega_{2}+\Omega_{0}$ & $0.104 \mathrm{D}-06$ & 1.74656 & \\
\hline-2.034 & 128.14122 & 2.3833729650 & $4 L_{3}-3 L_{4}$ & $0.290 \mathrm{D}-07$ & -0.04286 & \\
\hline-1.904 & 78.66637 & -3.1355655166 & $-7 L_{3}+8 L_{4}$ & $-0.811 \mathrm{D}-07$ & 0.11817 & \\
\hline-1.672 & 289.53911 & -0.5016928158 & $-L_{3}+L_{4}+\varpi_{4}$ & 0.319D-05 & -6.83233 & \\
\hline 1.670 & 287.32356 & 0.0006835386 & $-\rho-3 \varpi_{4}$ & $0.128 \mathrm{D}-06$ & -1.67808 & \\
\hline-1.543 & 356.32749 & -0.3750361593 & $-L_{4}+L_{\mathrm{S}}$ & $0.110 \mathrm{D}-06$ & & \\
\hline 1.336 & 252.77931 & -2.4221246132 & $-L_{1}+3 L_{4}-\varpi_{4}$ & $-0.104 \mathrm{D}-05$ & 2.21256 & \\
\hline 1.267 & 182.83092 & -0.3706858488 & $-L_{4}+4 L_{\mathrm{S}}$ & $0.350 \mathrm{D}-06$ & & \\
\hline 1.186 & 77.89774 & 1.1251084136 & $3 L_{4}-3 L_{\mathrm{S}}$ & $-0.265 \mathrm{D}-06$ & & \\
\hline 1.154 & 294.51269 & 7.1160095483 & $3 L_{1}-2 L_{2}$ & 0.189D-05 & -3.12397 & \\
\hline-1.051 & 195.48021 & -1.2539396667 & $-4 L_{3}+6 L_{4}-\varpi_{4}$ & $-0.669 \mathrm{D}-05$ & 14.27522 & \\
\hline 1.002 & 202.69555 & 0.0057685340 & $4 L_{\mathrm{S}}-\varpi_{4}$ & $0.136 \mathrm{D}-06$ & & \\
\hline-0.892 & 124.01796 & 0.0005884547 & $-\rho-\varpi_{3}-2 \varpi_{4}$ & $0.293 \mathrm{D}-08$ & -0.24496 & \\
\hline
\end{tabular}

Table 18. Solution for the variable $\zeta_{4}$ (inclination and node of Callisto). The series is expressed in complex exponentials.

\begin{tabular}{rrrcrr}
\hline \hline $\begin{array}{r}\text { Amplitude } \\
(\mathrm{km})\end{array}$ & $\begin{array}{c}\text { Phase } \\
(\mathrm{deg})\end{array}$ & $\begin{array}{c}\text { Frequency } \\
(\mathrm{rad} / \mathrm{day})\end{array}$ & Identification & $\begin{array}{r}\text { Freq. diff. } \\
(\mathrm{rad} / \mathrm{day})\end{array}$ & $\begin{array}{r}\text { Ph. diff. } \\
(\mathrm{deg})\end{array}$ \\
\hline 7235.560 & 138.27718 & 0.0000000000 & $\Omega_{0}$ & $0.000 \mathrm{D}+00$ & 0.00000 \\
4228.368 & 342.18035 & -0.0000305613 & $\Omega_{4}$ & $0.000 \mathrm{D}+00$ & 0.00000 \\
-490.458 & 191.18883 & -0.0001249131 & $\Omega_{3}$ & $0.291 \mathrm{D}-10$ & 0.00067 \\
62.541 & 318.58824 & 0.0029003769 & $2 L_{\mathrm{S}}$ & $-0.939 \mathrm{D}-08$ & \\
9.364 & 16.32255 & -0.0014500572 & $-L_{\mathrm{S}}$ & $-0.127 \mathrm{D}-06$ & \\
-9.306 & 60.02826 & -0.0005692030 & $\Omega_{2}$ & $-0.342 \mathrm{D}-08$ & 0.00089 \\
8.275 & 101.32342 & 0.0014501345 & $L_{\mathrm{S}}$ & $0.493 \mathrm{D}-07$ & \\
7.086 & 261.08358 & 0.0043504645 & $3 L_{\mathrm{S}}$ & $0.867 \mathrm{D}-07$ & \\
-5.804 & 115.13221 & 0.0029313051 & $2 L_{\mathrm{S}}-\Omega_{4}+\Omega_{0}$ & $-0.376 \mathrm{D}-06$ & \\
\hline
\end{tabular}

